\title{
Metastability of Kolmogorov flows and inviscid damping of shear flows
}

\author{
Zhiwu Lin \\ School of Mathematics \\ Georgia Institute of Technology \\ Atlanta, GA 30332, USA \\ Ming Xu \\ Department of Mathematics \\ JiNan University \\ Guangzhou, 510632, China
}

\begin{abstract}
First, we consider Kolmogorov flow (a shear flow with a sinusoidal velocity profile) for 2D Navier-Stokes equation on a torus. Such flows, also called bar states, have been numerically observed as one type of metastable states in the study of 2D turbulence. For both rectangular and square tori, we prove that the non-shear part of perturbations near Kolmogorov flow decays in a time scale much shorter than the viscous time scale. The results are obtained for both the linearized NS equations with any initial vorticity in $L^{2}$, and the nonlinear NS equation with initial $\mathrm{L}^{2}$ norm of vorticity of the size of viscosity. In the proof, we use the Hamiltonian structure of the linearized Euler equation and RAGE theorem to control the low frequency part of the perturbation. Second, we consider two classes of shear flows for which a sharp stability criterion is known. We show the inviscid damping in a time average sense for non-shear perturbations with initial vorticity in $L^{2}$. For the unstable case, the inviscid damping is proved on the center space. Our proof again uses the Hamiltonian structure of the linearized Euler equation and an instability index theory recently developed by Lin and Zeng for Hamiltonian PDEs.
\end{abstract}




\section{Introduction}

Consider 2D Navier-Stokes (NS) equation

$$
\partial_{t} U+U \cdot \nabla U-\nu \triangle U=-\nabla P
$$

on a torus

$$
\mathbb{T}_{\alpha}=\left\{0<y<2 \pi, 0<x<\frac{2 \pi}{\alpha}\right\}, \alpha>0,
$$

with the incompressible condition $\nabla \cdot U=0$, where $U=(u, v)$ is the fluid velocity and $\nu>0$ is the viscosity. More precisely, we impose the periodic boundary conditions

$$
U(0, y, t)=U(2 \pi / \alpha, y, t), U(x, 0, t)=U(x, 2 \pi, t) .
$$

The vorticity form of NS equation (1.1) is

$$
\omega_{t}+u \omega_{x}+v \omega_{y}-\nu \triangle \omega=0, \quad \omega=v_{x}-u_{y} .
$$

It is convenient to introduce the stream function $\psi$ such that $\omega=-\Delta \psi$ and $U=\nabla^{\perp} \psi=\left(\psi_{y},-\psi_{x}\right)$.

In the numerical and experimental study of $2 \mathrm{D}$ turbulence, it has often been observed ([17] [22] [6]) that the solutions to the two-dimensional Navier-Stokes (NS) equations with small viscosity rapidly approach certain long-lived coherent structures. Evidences also suggested that these quasistationary, or metastable solutions are closely related to stationary solutions of the inviscid Euler equations

$$
\omega_{t}+u \omega_{x}+v \omega_{y}=0, \quad \omega=v_{x}-u_{y}
$$

Since there is no forcing in (1.2), when $t \rightarrow \infty,\|\omega(t)\|_{L^{2}} \rightarrow 0$ in the viscous time scale $O\left(\frac{1}{\nu}\right)$, where $\omega(t)$ is the solution of $(1.2)$ with initial data $\omega(0) \in L^{2}$. We are interested in the dynamics of (1.2), particularly, the appearance and persistence of coherent states in the intermediate time scale $(0, T)$, where $1 \ll T \ll O\left(\frac{1}{\nu}\right)$. The first step is to prove that nearby solutions converge rapidly to these coherent states in a time scale $T \ll O\left(\frac{1}{\nu}\right)$. Such metastability problem is also called enhanced damping in the literature. Among the candidates of Euler steady solutions to explain the coherent structures, some authors (e.g. [6] [17] [22]) suggested that certain maximal entrophy solutions of the inviscid Euler equation are the most probable 
quasi-stationary that one would observe. The simplest of such maximal entrophy solutions is the Kolmogorov flow (also called bar states in [22]), that is, $u_{0}=(\sin y, 0)$ or $(\cos y, 0)$. The solution to (NS) with initial data $u_{0}$ is $u^{\nu}(t, y)=e^{-\nu t}(\sin y, 0)$. The linearized (NS) equation near $u^{\nu}$ is

$$
\partial_{t} \omega=\nu \Delta \omega-e^{-\nu t}\left[\sin y \partial_{x}\left(1+\Delta^{-1}\right)\right] \omega=L(t) \omega,
$$

where $\nu$ is the viscosity and $\omega$ is the vorticity perturbation. In [3], Beck and Wayne studied the following approximation of the linearized problem

$$
\partial_{t} \omega=\nu \Delta \omega-e^{-\nu t} \sin y \partial_{x} \omega=\tilde{L}(t) \omega,
$$

by dropping the nonlocal term $e^{-\nu t} \sin y \partial_{x} \Delta^{-1} \omega$ in (1.3). Define the following weighed $H^{1}$ space for non-shear vorticity functions

$$
\begin{aligned}
Z & =\left\{\sum_{k \neq 0} \omega=\omega_{k}(y) e^{i k x} \in L^{2}\right. \\
\|\omega\|_{Y}^{2} & \left.:=\sum_{k \neq 0}\left[\left\|\omega_{k}\right\|_{2}^{2}+\sqrt{\frac{\nu}{|k|}}\left\|\partial_{y} \omega_{k}\right\|_{2}^{2}+\frac{1}{\sqrt{\nu}|k|^{\frac{3}{2}}}\left\|C^{k} \omega_{k}\right\|_{2}^{2}\right]<\infty\right\},
\end{aligned}
$$

where $C^{k} \omega_{k}=-i k e^{\nu t}(\cos y) \omega_{k}$. It was proved in [3] that: For any $\tau>0$ and $T \in\left[0, \frac{\tau}{\nu}\right]$, there exist constants $K, M$ such that: if $\nu$ is small enough, then the solution to (1.4) with initial data $\omega(0) \in Z$ satisfies the estimate

$$
\|\omega(t)\|_{Z}^{2} \leq K e^{-M \sqrt{\nu} t}\|\omega(0)\|_{Z}^{2}, t \in[0, T] .
$$

The proof used Villani's hypocoercivity method ([21]). For the full linearized NS equation, numerical evidences in [3] suggest the same decay rate $O\left(e^{-\sqrt{\nu} t}\right)$.

In this paper, we study the full linearized equation (1.3) and the nonlinear equation (1.1) on a torus

$$
\mathbb{T}_{\alpha}=\left\{0<y<2 \pi, 0<x<\frac{2 \pi}{\alpha}\right\}
$$

with $\alpha \geq 1$, which is the sharp stability condition of Kolmogorov flows for the 2D Euler equation (see Lemma 4.1 of [14]). Our first result is about the enhanced damping for the linearized NS equation. 
Theorem 1.1 Consider the linearized NS equation (1.3) on $\mathbb{T}_{\alpha}$ with $\alpha \geq 1$. Define the non-shear vorticity space

$$
X=\left\{\omega \in L^{2} \mid \omega=\sum_{0 \neq k \in \mathbf{Z}} \omega_{k}(y) e^{i k \alpha x}\right\}
$$

i) (Rectangular torus) Consider $\alpha>1$. For any $\tau>0$ and $\delta>0$, if $\nu$ is small enough, then the solution $\omega(t)$ to (1.3) with non-shear initial data $\omega(0) \in X$ satisfies $\left\|\omega\left(\frac{\tau}{\nu}\right)\right\|_{L^{2}}<\delta\|\omega(0)\|_{L^{2}}$.

ii) (Square torus) Consider $\alpha=1$. Let $P_{1}$ be the orthogonal projection from the non-shear space

$$
X=\left\{\omega \in L^{2} \mid \omega=\sum_{k \neq 0} \omega_{k}(y) e^{i k x}\right\}
$$

to the space $W_{1}$ spanned by $\{\cos x, \sin x\}$. For any $\tau>0$ and $\delta>0$, if $\nu$ is small enough, then the solution to (1.3) with initial data $\omega(0) \in X$ satisfies

$$
\left\|\left(I-P_{1}\right) \omega\left(\frac{\tau}{\nu}\right)\right\|_{L^{2}}<\delta\left\|\left(I-P_{1}\right) \omega(0)\right\|_{L^{2}} .
$$

Since $\tau$ can be arbitrarily small, above result implies a much enhanced decay in the time scale $O\left(\frac{\tau}{\nu}\right)$ compared with the viscous time scale $O\left(\frac{1}{\nu}\right)$. For shear initial data $\omega(0)=\omega_{0}(y)$, the linearized NS equation (1.3) is reduced to the heat equation $\partial_{t} \omega=\nu \partial_{y y} \omega$ and there is no enhanced decay. On the square torus, there are two additional kernels $\{\cos x, \sin x\}$ of the operator $1+\Delta^{-1}$, which correspond to exact solutions $e^{-\nu t}\{\cos x, \sin x\}$ of the NavierStokes equations. These two additional kernels (so called anomalous modes in [3]) need to be removed for the enhanced damping to hold true.

For the nonlinear Navier-Stokes equation, we have the following result.

Theorem 1.2 Consider the nonlinear NS equation (1.1) on $\mathbb{T}_{\alpha}$ with $\alpha \geq 1$. Denote $P_{2}$ to be the projection of $L^{2}\left(\mathbb{T}_{\alpha}\right)$ to the subspace $W_{2}=\operatorname{span}\{\cos y, \sin y\}$.

i) (Rectangular torus) Suppose $\alpha>1$. There exist $d>0$, such that: for any $\tau>0$ and $\delta>0$, if $\nu$ is small enough, then any solution to (1.1) with initial data $\omega(0) \in L^{2}$ and

$$
\left\|\left(I-P_{2}\right) \omega(0)\right\|_{L^{2}} \leq d \nu
$$


satisfies

$$
\left\|P_{\neq 0} \omega\left(\frac{\tau}{\nu}\right)\right\|_{L^{2}}<\delta\left\|P_{\neq 0} \omega(0)\right\|_{L^{2}} .
$$

Here, $P_{\neq 0}$ is the projection of $L^{2}$ to the non-shear space $X$, that is,

$$
P_{\neq 0} \omega=\omega-\frac{\alpha}{2 \pi} \int_{0}^{\frac{2 \pi}{\alpha}} \omega d x .
$$

ii) (Square torus) Suppose $\alpha=1$. There exist $d>0$, such that: for any $\tau>0$ and $\delta>0$, if $\nu$ is small enough, then any solution to (1.1) with initial data $\omega(0) \in L^{2}$ and $\left\|\left(I-P_{2}\right) \omega(0)\right\|_{L^{2}} \leq d \nu$, satisfies

$$
\inf _{0 \leq t \leq \frac{\tau}{\nu}}\left\|\left(1-P_{1}\right) P_{\neq 0} \omega(t)\right\|_{L^{2}}<\delta\left\|P_{\neq 0} \omega(0)\right\|_{L^{2}} .
$$

Here, the projections $P_{1}, P_{\neq 0}$ are defined as above.

In the above theorem, the metastability of Kolmogorov flow is proved for perturbations of the size $\nu$. More precisely, it is shown that the nonshear part of the perturbation is reduced to a factor $\delta$ of the initial size before the time scale $\frac{\tau}{\nu}$, which is much smaller than the viscous time scale $\frac{1}{\nu}$. Moreover, by choosing the initial data to be smaller, we can ensure that $\left\|\left(I-P_{2}\right) \omega(t)\right\|_{L^{2}} \leq d \nu$ for all $t>0$, thus we can repeatedly use Theorem 1.2 to get the rapid decay of the non-shear part before the viscous time scale. We refer to Remark 3.1 for more details.

Next, we discuss some key ideas in the proof of Theorems 1.1 and 1.2. Our work is partly motivated by the work of Constantin et al. [9] for the linear reaction diffusion equation

$$
\partial_{t} \phi+v_{0} \cdot \nabla \phi-\nu \Delta \phi=0
$$

with an incompressible flow $v_{0}(x)$. In [9], the enhanced damping in the sense of Theorem 1.1 is proved under the assumption that the operator $v_{0} \cdot \nabla$ has no non-constant eigenfunction in $H^{1}$. Their proof is to consider the high and low frequency parts of the solution $\phi(t)$ separately. For the high frequency part (i.e. $\|\nabla \phi\|_{L^{2}} \approx N\|\phi\|_{L^{2}}$ for $N$ large), the enhanced damping is ensured by the energy dissipation law

$$
\partial_{t}\|\phi\|_{L^{2}}^{2}=-\nu\|\nabla \phi\|_{L^{2}}^{2}
$$


The lower frequency part is shown to converge to zero in the time average sense, by using the following RAGE Theorem for the unitary group $e^{i t L}$ with the self-adjoint generator $L=i v_{0} \cdot \nabla$.

Theorem (RAGE) [7] Let $L$ be a self-adjoint operator in a Hilbert space $H, P_{c}$ is the projection to the continuous spectrum space of $L$ and $B$ is any compact operator, then

$$
\frac{1}{T} \int_{0}^{T}\left\|B e^{i t L} P_{c} \psi\right\|_{H}^{2} d t \rightarrow 0, \text { when } T \rightarrow \infty .
$$

In the proof of enhanced damping, $B$ is taken to be the projection to the low frequency modes. Then the RAGE Theorem implies that the low frequency modes decay in the time average sense.

To apply these ideas to prove the enhanced damping for the linearized Navier-Stokes equation (1.3), there are a few difficulties to be overcome. First, for the equation (1.3), there is no obvious dissipation law as (1.10). We derive the following identity

$$
\frac{d}{d t} \int_{\mathbb{T}_{\alpha}}\left(|\omega|^{2}-|\nabla \psi|^{2}\right) d x d y=-2 \nu \int_{\mathbb{T}_{\alpha}}\left(|\nabla \omega|^{2}-|\omega|^{2}\right) d x d y,
$$

where $\psi=(-\Delta)^{-1} \omega$ is the stream function. When $\alpha>1$, the quadratic forms on both sides of (1.11) are positive definite for non-shear vorticity (i.e. $\omega \in X$ ). When $\alpha=1$, the positivity is still true in the space $X_{1}=$ $\left(I-P_{1}\right) X_{0}$. This provides a substitute of (1.10).

Second, even if we ignore the factor $e^{-\nu t}$ in (1.3), the linearized Euler operator $A=-\sin y \partial_{x}\left(1+\Delta^{-1}\right)$ is not anti-self-adjoint and the RAGE theorem cannot be applied directly to $e^{t A}$. An important observation is that $A$ can be written in the Hamiltonian form $A=J L$, where

$$
J=-\sin y \partial_{x}, \quad L=1+\Delta^{-1}
$$

are anti-selfadjoint and adjoint operators respectively in $L^{2}$. When $\alpha>1$, since $L=1+\Delta^{-1}>0$ on the non-shear space $X$, we can define a new inner product by $[\cdot, \cdot]=\langle L \cdot, \cdot\rangle$ on $X$, which is equivalent to the $L^{2}$ inner product. We observe that the operator $A$ is anti-selfadjoint in the space $(X,[\cdot, \cdot])$. Moreover, on the space $X$, the operator $A$ can be shown to have no embedded eigenvalues in the continuous spectra. Thus RAGE theorem can be applied to the semigroup $e^{t A}$ to show the decay of the low frequency 
part in the time average sense. The linear enhanced damping for (1.3) then follows similarly as in [9]. For the square torus $(\alpha=1)$, there are additional anomalous modes $\{\cos x, \sin x\}$ lying in $\operatorname{ker}\left(1+\Delta^{-1}\right)$. For any initial data $\omega(0) \in X$, we note that $\omega_{1}=\left(I-P_{1}\right) \omega$ satisfies the equation

$$
\partial_{t} \omega_{1}=\nu \Delta \omega_{1}-e^{-\nu t}\left[\left(I-P_{1}\right) \sin y \partial_{x}\left(1+\Delta^{-1}\right)\right] \omega_{1} .
$$

Let

$$
A_{1}=-\left(I-P_{1}\right) \sin y \partial_{x}\left(1+\Delta^{-1}\right)=\left(I-P_{1}\right) J L,
$$

then it can be checked that $A_{1}$ is anti-selfadjoint on the space $X_{1}=\left(I-P_{1}\right) X$ in the inner product $[\cdot, \cdot]=\langle L \cdot, \cdot\rangle$, where the positivity of $\left.L\right|_{X_{1}}$ is used. So applying the RAGE theorem to the semigroup $e^{t A_{1}}$ on $X_{1}$, we can again show that the low frequency part of $\left(I-P_{1}\right) e^{t J L} \omega(0)$ decays in the time average sense.

For the nonlinear NS equation (1.2), the evolution of the shear and nonshear parts are strongly coupled. For an initial perturbation $\omega(0)$ of size $O(\nu)$ in $L^{2}$, the interaction terms are controllable and the nonlinear enhanced damping (metastability) still holds true. On the square torus, the analysis for the nonlinear problem is more involved due to the anomalous modes. We decompose the perturbation into four parts lying in: $W_{1}=$ $\operatorname{span}\{\cos x, \sin x\}$ and its complementary subspace $W_{1}^{\perp}$ in the non-shear space, $W_{2}=\operatorname{span}\{\cos y, \sin y\}$ and its complementary subspace $W_{2}^{\perp}$ in the shear space. By carefully analyzing the interaction of these four parts, we can show that the interaction terms in the nonlinear term $U \cdot \nabla \omega$ are under control when $\|\omega(0)\|_{L^{2}}=O(\nu)$. As a result, we can still split the non-shear vorticity into the low and high frequency parts, and treat them separately as for the linearized equation. Then the nonlinear metastability can be proved. On the square torus, numerical evidences ([6] [5]) suggested that the dipole states of the form $\omega_{0}=\cos x+\cos y$ or $\sin x+\sin y$ appear more often in the long time dynamics of 2D Turbulence. The dipole flows are nonparallel and the enhanced damping problem is much more subtle to study. At the end of Section 3, we discuss some partial results and difficulties with the dipole states. In particular, in Proposition 3.1, we give a RAGE type theorem for the linearized Euler equation at dipoles.

In our proof of linear and nonlinear enhanced damping for Navier-Stokes equation, the Hamiltonian structures of the linearized Euler operator play an important role both in the derivation of the dissipation law (1.11) and in the 
control of the low frequency part. As a further application of these Hamiltonian structures, we consider the linear inviscid damping of more general shear flows $(U(y), 0)$. We study two classes of shear flows. One class is the flows without inflection points, which are spectrally stable by the classical Rayleigh criterion. The other class (called class $\mathcal{K}^{+}$) is the flows $U(y)$ with one inflection value $U_{s}$ and $-\frac{U^{\prime \prime}}{U-U_{s}}>0$. These two classes cover all the shear flows whose nonlinear stability in $L^{2}$ vorticity might be studied by the energyCasimir method (see Remark 4.3). The flows in the first class are nonlinearly stable for any $x$ period $2 \pi / \alpha$ and are minimizers of the energy-Casimir functional. The flows in the second class are stable only when $\alpha>\alpha_{\max }$ for some critical wave number $\alpha_{\max }$, and are maximizers of the energy-Casimir functional. These shear flows often appear as long lived coherent states in 2D turbulence. For example, the Kolmogorov flows which are in class $\mathcal{K}^{+}$.

In Theorem 4.1, we give a RAGE theorem on the non-shear subspace $X$ of $L^{2}$ for stable shear flows in the first class and in class $\mathcal{K}^{+}$with $\alpha>\alpha_{\max }$. As a consequence, the decay of velocity (in the time average sense) is proved for any non-shear initial data with $L^{2}$ vorticity. Another consequence is the decay of low frequency modes in the $L^{2}$ norm of vorticity, which gives a justification of the dual cascade of 2D turbulence in a weak sense (see Remark 4.2). For the critical case $\alpha=\alpha_{\max }$, the linearized Euler operator $J L$ (defined in (1.12)) has zero as an embedded eigenvalue due to the nontrivial ker $L$. This case is very similar to the case of bar states on the square torus and can be treated similarly. The linear damping can be obtained by projecting out ker $L$.

The flows in class $\mathcal{K}^{+}$are unstable when $\alpha<\alpha_{\max }$ ([10]). Moreover, by using an instability index theory recently developed in [15] for Hamiltonian PDEs, we give an exact counting formula (Proposition 4.1) for the dimension of unstable modes of the linearized Euler equation. A corollary of this formula is that $\left.L\right|_{E^{c}} \geq 0$, where $E^{c}$ is the center space corresponding to the spectra of the linearized Euler operator $J L$ (defined in (1.12)) on the imaginary axis. Then the RAGE theorem, and as a consequence the damping of velocity, are obtained for the linearized Euler equation on $E^{c}$.

The inviscid damping was first known for the Couette flow in the 1907 work of Orr ([18]). In recent years, the inviscid damping phenomena attracted new attention. In [13], it was showed that if we consider initial (vorticity) perturbation in the Sobolev space $H^{s}\left(s<\frac{3}{2}\right)$ then the nonlinear damping is not true due to the existence of nonparallel steady flows of the form of 
Kelvin's cats eye near Couette. In [4], nonlinear inviscid damping was proved for perturbations near Couette in Gevrey class (i.e. almost analytic).

The linear inviscid damping for more general shear flows was also recently studied by some authors. Monotone shears were considered in [25] for the case near Couette, and in [23] for the more general case. The optimal decay rates $O(1 / t), O\left(1 / t^{2}\right)$ for the horizontal and vertical velocities were obtained for initial vorticity in $H^{1}$ and $H^{2}$ respectively. In [24], general shear flows satisfying some nondegeneracy conditions were considered, and certain spacetime estimates for velocities of the linearized Euler equation were obtained for initial vorticity in $H^{1}$. The optimal decay rates were also obtained in [24] for a special class of symmetric shear flows. The non-existence of embedded eigenvalues was assumed in above works.

We comment on some differences of our results on inviscid damping with the previous work. First, for the two classes of shear flows we considered, we do not need to assume the non-existence of embedded eigenvalues. This assumption is proved to be true for flows without inflection points and for flows in class $\mathcal{K}^{+}$with $\alpha>\alpha_{\max }$. But for $\alpha=\alpha_{\max }$ and some $\alpha<\alpha_{\max }$, zero is indeed an embedded eigenvalue. For these cases, the inviscid damping can still be proved as in Corollary 4.1 ii) and Theorem 4.2 ii), as well as for the Kolmogorov flow on the square torus. Second, the inviscid damping results we obtained are for the initial vorticity in $L^{2}$. In [23] [24], initial vorticity with higher regularity was considered and the linear damping for $L^{2}$ vorticity was not studied. In [23] [24] [25], the linearized Euler equation was studied in a channel. Here, we treat the cases of the channel and tori in a unified way. In some sense, the RAGE theorem type results imply more information than just the damping of velocities. For example, the decay of low frequency part of the vorticity does not follow from the decay of velocity. Third, our approach, which exploits the Hamiltonian structures of the Euler equation, does not rely on ODE techniques. Therefore, it could be used for the problems involving nonparallel flows, see Proposition 3.1 for dipoles and Theorem 11.7 in [15] for general steady Euler flows. Moreover, more information on the damping could be derived from the regularity properties of the spectral measure of $J L$ (see Remark 4.1). This might provide an alternative approach to study the inviscid damping in other problems.

This paper is organized as follows. In Section 2, we study the linear enhanced damping for the linearized Navier-Stokes equation. In Section 3, the nonlinear enhanced damping (i.e. metastability of Kolmogorov flows) is proved for the nonlinear Navier-Stokes equation. We discuss the cases of 
rectangular and square tori separately in Sections 2 and 3. In Section 4, the linear inviscid damping is proved for both stable and unstable shear flows.

\section{Linear enhanced damping}

In this section, we prove Theorem 1.1 on the enhanced damping for the linearized Navier-Stokes equation (1.3). We consider the cases of rectangular and square tori separately.

\subsection{Linearized Navier-Stokes on a rectangular torus}

Consider the linearized equation (1.3) on a torus

$$
\mathbb{T}_{\alpha}=\left\{0<y<2 \pi, 0<x<\frac{2 \pi}{\alpha}\right\}, \alpha>1 .
$$

We divide the proof of Theorem 1.1 i) into several steps. In the proof, we shall use $C$ to denote a generic constant in the estimates. First, we prove the dissipation law (1.11).

Lemma 2.1 Let $\omega(t)$ be a solution of (1.3) with the initial data $\omega(t) \in$ $L^{2}\left(\mathbb{T}_{\alpha}\right)$. Then

$$
\frac{d}{d t} \int_{\mathbb{T}_{\alpha}}\left(|\omega|^{2}-|\nabla \psi|^{2}\right) d x d y=-2 \nu \int_{\mathbb{T}_{\alpha}}\left(|\nabla \omega|^{2}-|\omega|^{2}\right) d x d y,
$$

for any $t>0$.

Proof. The equation (1.3) can be written as

$$
\partial_{t} \omega=\nu \Delta \omega+e^{-\nu t} J L \omega,
$$

where $J, L$ are defined in (1.12). Thus we have

$$
\begin{aligned}
\frac{d}{d t} \int_{\mathbb{T}_{\alpha}}\left(|\omega|^{2}-|\nabla \psi|^{2}\right) d x d y & =\frac{d}{d t}\langle L \omega, \omega\rangle=2\left\langle L \omega, \omega_{t}\right\rangle \\
& =e^{-\nu t}\langle L \omega, J L \omega\rangle+2 \int_{\mathbb{T}_{\alpha}} \nu \Delta \omega(\omega-\psi) d x d y \\
& =-2 \nu \int_{\mathbb{T}_{\alpha}}\left(|\nabla \omega|^{2}-|\omega|^{2}\right) d x d y .
\end{aligned}
$$


In the last equality above, we use integration by parts and the fact that $J$ is anti-selfadjoint.

In the next lemma, we show that the quadratic forms on both sides of (2.1) are positive definite for a non-shear vorticity $\omega$.

Lemma 2.2 Let $\alpha>1$ and $\omega \in X \cap H^{1}\left(\mathbb{T}_{\alpha}\right)$. Then there exists a constant $c_{0}>0$ depending only on $\alpha$ such that

$$
\int_{\mathbb{T}_{\alpha}}\left(|\omega|^{2}-|\nabla \psi|^{2}\right) d x d y \geq c_{0}\|\omega\|_{L^{2}}^{2}
$$

and

$$
\int_{\mathbb{T}_{\alpha}}\left(|\nabla \omega|^{2}-|\omega|^{2}\right) d x d y \geq c_{0}\|\omega\|_{H^{1}}^{2}
$$

Proof. For any

$$
\omega=\sum_{k \neq 0} \omega_{k}(y) e^{i k \alpha x} \in H^{1}\left(\mathbb{T}_{\alpha}\right)
$$

we have

$$
\begin{aligned}
\int_{\mathbb{T}_{\alpha}}\left(|\omega|^{2}-|\nabla \psi|^{2}\right) d x d y & =\sum_{0 \neq k \in \mathbf{Z}}\left\langle\left(1-\left(-\frac{d^{2}}{d y^{2}}+\alpha^{2} k^{2}\right)^{-1}\right) \omega_{k}, \omega_{k}\right\rangle \\
& \geq\left(1-\alpha^{-2}\right) \sum_{0 \neq k \in \mathbf{Z}}\left(\omega_{k}, \omega_{k}\right)=\left(1-\alpha^{-2}\right)\|\omega\|_{L^{2}}^{2},
\end{aligned}
$$

and

$$
\begin{aligned}
\int_{\mathbb{T}_{\alpha}}\left(|\nabla \omega|^{2}-|\omega|^{2}\right) d x d y & =\sum_{0 \neq k \in \mathbf{Z}}\left\langle\left(-\frac{d^{2}}{d y^{2}}+\alpha^{2} k^{2}-1\right) \omega_{k}, \omega_{k}\right\rangle \\
& \geq \sum_{0 \neq k \in \mathbf{Z}} \int\left|\omega_{k}^{\prime}(y)\right|^{2} d y+\left(\alpha^{2}-1\right) \int\left|\omega_{k}(y)\right|^{2} d y \\
& \geq \min \left\{1, \alpha^{2}-1\right\}\|\omega\|_{H^{1}}^{2} .
\end{aligned}
$$

Next, we study the linearized Euler equation at the Kolmogorov flow

$$
\partial_{t} \omega=-\sin y \partial_{x}\left(1+\Delta^{-1}\right) \omega=J L \omega
$$


Lemma 2.3 Let $\omega(t)$ be a solution of (2.4) with $\omega(0) \in X \cap H^{1}\left(\mathbb{T}_{\alpha}\right)$. Then

$$
\|\omega(t)\|_{H^{1}} \leq C(1+t)\|\omega(0)\|_{H^{1}},
$$

for some constant $C$.

Proof. First, we note that

$$
\langle L \omega, \omega\rangle=\int_{\mathbb{T}_{\alpha}}\left(|\omega|^{2}-|\nabla \psi|^{2}\right) d x d y
$$

is conserved for (1.3). Therefore by the positivity estimate (2.2), we have

$$
\|\omega(t)\|_{L^{2}}=\left\|e^{t J L} \omega(0)\right\|_{L^{2}} \leq C\|\omega(0)\|_{L^{2}},
$$

for some constant $C$. Taking $\partial_{x}$ of (2.4), we have

$$
\partial_{t} \partial_{x} \omega=-\sin y \partial_{x}\left(1+\Delta^{-1}\right) \partial_{x} \omega
$$

and therefore

$$
\left\|\partial_{x} \omega(t)\right\|_{L^{2}}=\left\|e^{t J L} \partial_{x} \omega(0)\right\|_{L^{2}} \leq C\left\|\partial_{x} \omega(0)\right\|_{L^{2}} .
$$

Taking $\partial_{y}$ of (2.4), we have

$$
\partial_{t} \partial_{y} \omega=-\sin y \partial_{x}\left(1+\Delta^{-1}\right) \partial_{y} \omega-\cos y \partial_{x}\left(1+\Delta^{-1}\right) \omega,
$$

and

$$
\partial_{y} \omega(t)=e^{t J L} \partial_{y} \omega(0)-\int_{0}^{t} e^{(t-s) J L} \cos y \partial_{x}\left(1+\Delta^{-1}\right) \omega(s) d s .
$$

Therefore

$$
\begin{aligned}
\left\|\partial_{y} \omega(t)\right\|_{L^{2}} & \leq C\left(\left\|\partial_{y} \omega(0)\right\|_{L^{2}}+\int_{0}^{t}\left\|\partial_{x}\left(1+\Delta^{-1}\right) \omega(s)\right\|_{L^{2}} d s\right) \\
& \leq C(1+t)\|\nabla \omega(0)\|_{L^{2}} .
\end{aligned}
$$

This finishes the proof of the lemma.

In the next lemma, we study the spectral properties of the linearized Euler operator $A=J L$. 
Lemma 2.4 i) The operator $A: X^{*} \rightarrow X$ is anti-selfadjoint in the inner product $[\cdot, \cdot]=\langle L \cdot, \cdot\rangle$.

ii) The spectrum of $A$ lies on the imaginary axis and is purely continuous.

Proof. i) By the positivity of $L$ on $X,[\cdot, \cdot]=\langle L \cdot, \cdot\rangle$ defines an equivalent inner product to the $L^{2}$ inner product. For any $\omega_{1}, \omega_{2} \in X$, we have

$$
\begin{aligned}
{\left[A \omega_{1}, \omega_{2}\right] } & =\left\langle L J L \omega_{1}, \omega_{2}\right\rangle=\left\langle J L \omega_{1}, L \omega_{2}\right\rangle=-\left\langle L \omega_{1}, J L \omega_{2}\right\rangle \\
& =-\left[\omega_{1}, A \omega_{2}\right],
\end{aligned}
$$

and thus $A$ is anti-selfadjoint on $(X,[\cdot, \cdot])$.

ii) By property i), the spectrum of $A$ in $L^{2}$ is on the imaginary axis. Since $A=-\sin y \partial_{x}\left(1+\Delta^{-1}\right)$ is a compact perturbation of $D=-\sin y \partial_{x}$, by Weyl's Theorem the continuous spectrum of $A$ is the same as that of $D$, which is clearly the whole imaginary axis. It remains to show that there is no embedded eigenvalue of $A$ on the imaginary axis. Suppose $A \omega=\lambda \omega$, where $\lambda \in i \mathbf{R}$ and $0 \neq \omega \in X$. Let

$$
\omega=\sum_{0 \neq k \in \mathbf{Z}} \omega_{k}(y) e^{i k \alpha x}, \quad \psi_{k}=\left(-\frac{d^{2}}{d y^{2}}+\alpha^{2} k^{2}\right)^{-1} \omega_{k} .
$$

Then if $\omega_{k} \neq 0$, we have

$$
i k \alpha \sin y\left(\omega_{k}-\psi_{k}\right)=\lambda \omega_{k}
$$

which is equivalent to the Rayleigh equation

$$
\left(-\frac{d^{2}}{d y^{2}}+\alpha^{2} k^{2}-\frac{\sin y}{\sin y-c}\right) \psi_{k}=0
$$

with $c=\frac{\lambda}{i k \alpha} \in \mathbf{R}$. Since $\omega_{k} \in L^{2}$ implies $\psi_{k} \in H^{2}$, by Lemma 5.1 in the Appendix, we must have $c=0$ which is the only inflection value of $\sin y$. Thus

$$
\left(-\frac{d^{2}}{d y^{2}}+\alpha^{2} k^{2}-1\right) \psi_{k}=0
$$

which implies that $\psi_{k}=0$ since $\alpha>1$. This contradiction rules out the embedded imaginary eigenvalues of $A$ in $X$.

By the above Lemma and the RAGE theorem, we have 
Corollary 2.1 Let $B$ be any compact operator in $L^{2}\left(\mathbb{T}_{\alpha}\right)$. Then

$$
\frac{1}{T} \int_{0}^{T}\|B \omega(t)\|_{L^{2}}^{2} d t \rightarrow 0, \text { when } T \rightarrow \infty
$$

for any solution $\omega(t)$ of (2.4) with $\omega(0) \in X$.

For the proof of the enhanced damping, we need a more quantitative version of RAGE theorem. Let $\alpha^{2} \leq \lambda_{1} \leq \lambda_{2} \leq \ldots$ be the eigenvalues of the operator $-\triangle$ on $X$ and $e_{1}, e_{2} \ldots$ be the corresponding orthonormal eigenvectors. Let $P_{N}$ denote the orthogonal projection on the subspace spanned by the first $N$ eigenvectors $e_{1}, e_{2}, \ldots, e_{N}$ and $S=\left\{\omega \in X:\|\omega\|_{L^{2}}=1\right\}$ be the unit sphere in $X$. Denote the norms

$$
\begin{gathered}
\|\omega\|_{X}^{2}=\left\langle\left(1+\Delta^{-1}\right) \omega, \omega\right\rangle=\int_{\mathbb{T}_{\alpha}}\left(|\omega|^{2}-|\nabla \psi|^{2}\right) d x d y, \\
\|\omega\|_{X^{1}}^{2}=\langle(-\Delta-1) \omega, \omega\rangle=\int_{\mathbb{T}_{\alpha}}\left(|\nabla \omega|^{2}-|\omega|^{2}\right) d x d y
\end{gathered}
$$

which are equivalent to $L^{2}$ and $H^{1}$ norms on $X$. Let $\omega=\sum_{k \geq 1} c_{k} e_{k}$, then

$$
\|\omega\|_{X}^{2}=\sum_{k \geq 1}\left(1-\frac{1}{\lambda_{k}}\right)\left|c_{k}\right|^{2}
$$

and

$$
\|\omega\|_{X^{1}}^{2}=\sum_{k \geq 1}\left(\lambda_{k}-1\right)\left|c_{k}\right|^{2} .
$$

The following version of the RAGE theorem is obtained as in [9].

Lemma 2.5 Let $K \subset S$ be a compact set and $J, L$ are defined in (1.12). For any $N, \kappa>0$, there exists $T_{c}(N, \kappa, K)$ such that for all $T \geq T_{c}$ and any $\omega(0) \in K$,

$$
\frac{1}{T} \int_{0}^{T}\left\|P_{N} e^{t J L} \omega(0)\right\|_{X}^{2} d t \leq \kappa\|\omega(0)\|_{X}^{2} .
$$

Now we estimate the difference of the solutions of the linearized NS and Euler equations. 
Lemma 2.6 Let $\omega^{\nu}, \omega^{0}$ be the solutions of the linearized NS equation (1.3) and Euler equation (2.4) with the initial data in $X \cap H^{1}$. Then there exists some constant $C_{0}>0$ such that

$$
\frac{d}{d t}\left\|\omega^{\nu}-\omega^{0}\right\|_{X}^{2} \leq C_{0} \nu\left(1+t^{2}\right)\left\|\omega^{0}(t)\right\|_{H^{1}}^{2}
$$

for all $t \in(0,+\infty)$.

Proof. Let $\psi^{\nu}, \psi^{0}$ be the corresponding stream functions. Denote $\omega=$ $\omega^{\nu}-\omega^{0}$ and $\psi=\psi^{\nu}-\psi^{0}$, then

$$
\omega_{t}+e^{-\nu t} \sin y \partial_{x}(\omega-\psi)+\left(e^{-\nu t}-1\right) \sin y \partial_{x}\left(\omega^{0}-\psi^{0}\right)-\nu \triangle \omega^{\nu}=0 .
$$

We have

$$
\begin{aligned}
& \frac{d}{d t} \frac{1}{2}\left\|\omega^{\nu}-\omega^{0}\right\|_{X}^{2}=\frac{d}{d t} \frac{1}{2} \int_{\mathbb{T}_{\alpha}}\left(|\omega|^{2}-|\nabla \psi|^{2}\right) d x d y=\int_{\mathbb{T}_{\alpha}} \omega_{t}(\omega-\psi) d x d y \\
=- & \int_{\mathbb{T}_{\alpha}}\left(e^{-\nu t}-1\right) \sin y \partial_{x}\left(\omega^{0}-\psi^{0}\right)(\omega-\psi) d x d y+\nu \int_{\mathbb{T}_{\alpha}} \triangle \omega^{\nu}\left(\omega^{\nu}-\psi^{\nu}\right) d x d y \\
& \quad-\nu \int_{\mathbb{T}_{\alpha}} \triangle \omega^{\nu}\left(\omega^{0}-\psi^{0}\right) d x d y \\
= & I+I I+I I I .
\end{aligned}
$$

Since $0 \leq 1-e^{-\nu t} \leq \nu t$ when $t>0$, we have

$$
\begin{aligned}
I & =\left(1-e^{-\nu t}\right) \int_{\mathbb{T}_{\alpha}} \sin y \partial_{x}\left(\omega^{0}-\psi^{0}\right)\left(\omega^{\nu}-\psi^{\nu}\right) d x d y \\
& =-\left(1-e^{-\nu t}\right) \int_{\mathbb{T}_{\alpha}} \sin y \partial_{x}\left(\omega^{\nu}-\psi^{\nu}\right)\left(\omega^{0}-\psi^{0}\right) d x d y \\
& \leq C \nu t\left\|\omega^{\nu}\right\|_{H^{1}}\left\|\omega^{0}\right\|_{L^{2}} .
\end{aligned}
$$

By integration by parts and (2.3),

$$
I I=-\nu \int_{\mathbb{T}_{\alpha}}\left(\left|\nabla \omega^{\nu}\right|^{2}-\left|\omega^{\nu}\right|^{2}\right) d x d y \leq-c_{0} \nu\left\|\omega^{\nu}\right\|_{H^{1}}^{2} .
$$

For the last term, we have

$$
I I I=\nu \int_{\mathbb{T}_{\alpha}} \nabla \omega^{\nu} \cdot \nabla\left(\omega^{0}-\psi^{0}\right) d x d y \leq \nu\left\|\omega^{\nu}\right\|_{H^{1}}\left\|\omega^{0}\right\|_{H^{1}} .
$$


Combining above, we get

$$
\begin{aligned}
& \frac{d}{d t} \frac{1}{2} \int_{\mathbb{T}_{\alpha}}\left(|\omega|^{2}-|\nabla \psi|^{2}\right) d x d y \\
\leq & \nu\left(-c_{0}\left\|\omega^{\nu}\right\|_{H^{1}}^{2}+\left\|\omega^{\nu}\right\|_{H^{1}}\left(C t\left\|\omega^{0}\right\|_{L^{2}}+\left\|\omega^{0}\right\|_{H^{1}}\right)\right) \\
\leq & C \nu\left(1+t^{2}\right)\left\|\omega^{0}(t)\right\|_{H^{1}}^{2} .
\end{aligned}
$$

This proves (2.6).

As a corollary, combining (2.6), Lemma 2.3 and (2.3), we have

$$
\left\|\omega^{\nu}(t)-\omega^{0}(t)\right\|_{X}^{2} \leq\left\|\omega^{\nu}(0)-\omega^{0}(0)\right\|_{X}^{2}+C_{1} \nu\left(1+t^{5}\right)\left\|\omega^{0}(0)\right\|_{X^{1}}^{2},
$$

for some constant $C_{1}>0$.

We are now ready to prove the linear enhanced damping.

Proof of Theorem $1.1 \mathrm{i}$ ). The proof follows by the same arguments in [9]. We sketch it here. Fixed $\delta, \tau>0$, we choose $N$ such that

$$
\exp \left(-2 \lambda_{N} \tau\right)<c_{0} \delta^{2}
$$

where $c_{0}$ is the constant in (2.2). Define a compact set

$$
K=\operatorname{span}\left\{e_{1}, \cdots, e_{N}\right\}=R\left(P_{N}\right) .
$$

Denote $t_{1}=T_{c}\left(N, \frac{1}{10}, K\right)$ as in Lemma 2.5 and let $\nu(\delta, \tau)$ be such that

$$
\nu(\delta, \tau) C_{1}\left(1+t_{1}^{5}\right)<\frac{1}{10 \lambda_{N}},
$$

where $C_{1}$ is the constant in the estimate (2.7). For $0<\nu<\nu(\delta, \tau)$, suppose that

$$
\left\|\omega^{\nu}(t)\right\|_{X^{1}}^{2}>\lambda_{N}\left\|\omega^{\nu}(t)\right\|_{X}^{2}
$$

is true for $t$ in some interval $(a, b) \subset(0, \tau / \nu)$. Then by $(2.1)$, we have

$$
\left\|\omega^{\nu}(b)\right\|_{X}^{2} \leq \exp \left(-\nu \lambda_{N}(b-a)\right)\left\|\omega^{\nu}(a)\right\|_{X}^{2}
$$

Now consider any $t_{0} \in(0, \tau / \nu)$ satisfying

$$
\left\|\omega^{\nu}\left(t_{0}\right)\right\|_{X^{1}}^{2} \leq \lambda_{N}\left\|\omega^{\nu}\left(t_{0}\right)\right\|_{X}^{2} .
$$


Denote $\omega_{0}=\omega^{\nu}\left(t_{0}\right)$ and let $\omega^{0}(t)\left(t \in\left[t_{0}, t_{0}+t_{1}\right]\right)$ be the solution of $(2.4)$ with $\omega^{0}\left(t_{0}\right)=\omega_{0}$. By the choice of $t_{0}, \nu(\delta, \tau)$ and $(2.7)$, we have

$$
\left\|\omega^{\nu}(t)-\omega^{0}(t)\right\|_{X}^{2} \leq \frac{1}{10}\left\|\omega_{0}\right\|_{X}^{2}, \forall t \in\left[t_{0}, t_{0}+t_{1}\right] .
$$

By the definition of $t_{1}$, we have

$$
\frac{1}{t_{1}} \int_{t_{0}}^{t_{0}+t_{1}}\left\|P_{N} \omega^{0}(t)\right\|_{X}^{2} d t \leq \frac{1}{10}\left\|\omega_{0}\right\|_{X}^{2}
$$

Since $\left\|\omega^{0}(t)\right\|_{X}=\left\|\omega_{0}\right\|_{X}$ by the conservation of $\langle L \omega, \omega\rangle$ for the equation (2.4), it follows that

$$
\frac{1}{t_{1}} \int_{t_{0}}^{t_{0}+t_{1}}\left\|\left(1-P_{N}\right) \omega^{0}(t)\right\|_{X}^{2} d t \geq \frac{9}{10}\left\|\omega_{0}\right\|_{X}^{2} .
$$

Combined with (2.9), above implies that

$$
\frac{1}{t_{1}} \int_{t_{0}}^{t_{0}+t_{1}}\left\|\left(1-P_{N}\right) \omega^{\nu}(t)\right\|_{X}^{2} d t \geq \frac{1}{2}\left\|\omega_{0}\right\|_{X}^{2} .
$$

For $\omega^{\nu}(t)=\sum_{k \geq 1} c_{k} e_{k}$, we have

$$
\begin{aligned}
\left\|\omega^{\nu}(t)\right\|_{X^{1}}^{2} & =\sum_{k \geq 1}\left(\lambda_{k}-1\right)\left|c_{k}\right|^{2} \geq \frac{1}{\lambda_{N}} \sum_{k \geq N+1}\left(1-\frac{1}{\lambda_{k}}\right)\left|c_{k}\right|^{2} \\
& =\frac{1}{\lambda_{N}}\left\|\left(1-P_{N}\right) \omega^{\nu}(t)\right\|_{X}^{2}
\end{aligned}
$$

and thus

$$
\int_{t_{0}}^{t_{0}+t_{1}}\left\|\omega^{\nu}(t)\right\|_{X^{1}}^{2} d t \geq \frac{\lambda_{N} t_{1}}{2}\left\|\omega_{0}\right\|_{X}^{2}=\frac{\lambda_{N} t_{1}}{2}\left\|\omega^{\nu}\left(t_{0}\right)\right\|_{X}^{2} .
$$

Then (2.1) implies that

$$
\begin{aligned}
\left\|\omega^{\nu}\left(t_{0}+t_{1}\right)\right\|_{X}^{2} & \leq\left\|\omega^{\nu}\left(t_{0}\right)\right\|_{X}^{2}-2 \nu \int_{t_{0}}^{t_{0}+t_{1}}\left\|\omega^{\nu}(t)\right\|_{X^{1}}^{2} d t \\
& \leq\left(1-\lambda_{N} \nu t_{1}\right)\left\|\omega^{\nu}\left(t_{0}\right)\right\|_{X}^{2} \leq e^{-\lambda_{N} \nu t_{1}}\left\|\omega^{\nu}\left(t_{0}\right)\right\|_{X}^{2} .
\end{aligned}
$$


We can split the interval $\left[0, \frac{\tau}{\nu}\right]$ into a union of intervals such that either $(2.8)$ or (2.10) holds true. Therefore we have

$$
\left\|\omega^{\nu}\left(\frac{\tau}{\nu}\right)\right\|_{X}^{2} \leq e^{-\lambda_{N} \tau}\left\|\omega^{\nu}(0)\right\|_{X}^{2}<c_{0} \delta^{2}\left\|\omega^{\nu}(0)\right\|_{X}^{2}
$$

and by $(2.2)$

$$
\left\|\omega^{\nu}\left(\frac{\tau}{\nu}\right)\right\|_{L^{2}}^{2} \leq \frac{1}{c_{0}}\left\|\omega^{\nu}\left(\frac{\tau}{\nu}\right)\right\|_{X}^{2}<\delta^{2}\left\|\omega^{\nu}(0)\right\|_{X}^{2}<\delta^{2}\left\|\omega^{\nu}(0)\right\|_{L^{2}}^{2} .
$$

This finishes the proof of Theorem $1.1 \mathrm{i}$ ).

\subsection{Linearized Navier-Stokes on a square torus}

Now we consider the linearized equation (1.3) on the square torus

$$
\mathbb{T}=\{0<y<2 \pi, 0<x<2 \pi\}
$$

In this case, there is a two dimensional kernel space $W_{1}$ spanned by $\{\cos x, \sin x\}$ of the operator $L=1+\Delta^{-1}$ on the non-shear space $X$. We will sketch the changes induced by these anomalous modes, in the proof of Theorem $1.1 \mathrm{ii}$ ).

First, we note that $L$ is positive on the space $X_{1}=\left(I-P_{1}\right) X$, where $P_{1}$ is the projection of $X$ to $W_{1}$. Let $\omega(t)$ be the solution of (1.3) with any initial data $\omega(0) \in X$. Then $\omega_{1}=\left(I-P_{1}\right) \omega$ satisfies the equation

$$
\partial_{t} \omega_{1}=\nu \Delta \omega_{1}-e^{-\nu t}\left[\left(I-P_{1}\right) \sin y \partial_{x}\left(1+\Delta^{-1}\right)\right] \omega_{1} .
$$

It is easy to check that the same dissipation law

$$
\frac{d}{d t} \int_{\mathbb{T}}\left(\left|\omega_{1}\right|^{2}-\left|\nabla \psi_{1}\right|^{2}\right) d x d y=-2 \nu \int_{\mathbb{T}}\left(\left|\nabla \omega_{1}\right|^{2}-\left|\omega_{1}\right|^{2}\right) d x d y,
$$

holds true for (2.11). Moreover, there exists $c_{0}>0$ such that

$$
\int_{\mathbb{T}}\left(|\omega|^{2}-|\nabla \psi|^{2}\right) d x d y \geq c_{0}\|\omega\|_{L^{2}}^{2}
$$

and

$$
\int_{\mathbb{T}}\left(|\nabla \omega|^{2}-|\omega|^{2}\right) d x d y \geq c_{0}\|\omega\|_{H^{1}}^{2}
$$


for $\omega \in X_{1}$. Define the operator $A_{1}:\left(X_{1}\right)^{*} \rightarrow X_{1}$ by

$$
A_{1}=-\left(I-P_{1}\right) \sin y \partial_{x}\left(1+\Delta^{-1}\right)=\left(I-P_{1}\right) J L .
$$

Since $\left.L\right|_{X_{1}}>0,[\cdot, \cdot]=\langle L \cdot, \cdot\rangle$ is again an equivalent inner product on $X_{1}$ and $A_{1}$ is anti-selfadjoint on $\left(X_{1},[\cdot, \cdot]\right)$. Indeed, for any $w_{1}, w_{2} \in X_{1}$

$$
\begin{aligned}
{\left[A_{1} w_{1}, w_{2}\right] } & =\left\langle L\left(I-P_{1}\right) J L w_{1}, w_{2}\right\rangle=\left\langle L J L w_{1}, w_{2}\right\rangle=-\left\langle L w_{1}, J L w_{2}\right\rangle \\
& =-\left\langle L w_{1},\left(I-P_{1}\right) J L w_{2}\right\rangle=-\left[w_{1}, A_{1} w_{2}\right] .
\end{aligned}
$$

Therefore the spectrum of $A_{1}$ lies on the imaginary axis. We will show that $A_{1}$ has no embedded imaginary eigenvalues.

Lemma 2.7 The spectrum of $A_{1}$ is purely continuous.

Proof. Suppose $A_{1}$ has an eigenvalue $\lambda \in i \mathbf{R}$ and $A_{1} \omega=\lambda \omega$, where $0 \neq \omega \in X_{1}$. Then

$$
A \omega-\lambda \omega=J L \omega-\lambda \omega=\tilde{\omega} \in W_{1} .
$$

If $\lambda \neq 0$, by noting that $A \tilde{\omega}=0$, we get

$$
A\left(\omega+\frac{1}{\lambda} \tilde{\omega}\right)=\lambda\left(\omega+\frac{1}{\lambda} \tilde{\omega}\right) .
$$

That is, $\lambda$ is an eigenvalue of $A$. This is a contradiction, since by the proof of Lemma 2.4, the operator $A$ has no nonzero eigenvalues. If $\lambda=0$, then we must have $A \omega=\tilde{\omega}$ for some $0 \neq \tilde{\omega} \in W_{1}$, since $\omega \in X_{1}$ implies that $A \omega \neq 0$. Let $\tilde{\omega}=c_{1} e^{i x}+c_{-1} e^{-i x} \in W_{1}$ and

$$
\psi=\Delta^{-1} \omega=a_{1}(y) e^{i x}+a_{-1}(y) e^{-i x} .
$$

From the equation $A \omega=\sin y \partial_{x}(\omega+\psi)=\tilde{\omega}$, we get

$$
a_{1}^{\prime \prime}(y)=\frac{c_{1}}{i \sin y}, \quad a_{-1}^{\prime \prime}(y)=\frac{c_{-1}}{-i \sin y},
$$

and thus $\psi \notin H^{2}$. This shows that 0 is not an eigenvalue of $A_{1}$ and the proof of the lemma is finished.

By the above lemma, we can use the RAGE theorem for the semigroup $e^{t A_{1}}$ on $X_{1}$, which corresponds to solutions of the projected linearized Euler equation

$$
\partial_{t} \omega=\left(I-P_{1}\right) \sin y \partial_{x}\left(1+\Delta^{-1}\right) \omega
$$


In particular, let $P_{N}$ be the projection of $L^{2}$ to the space spanned by the first $N$ eigenfunction of $-\triangle$ on $X_{1}$, then we have: For any $N, \kappa>0$, there exists $T_{c}(N, \kappa, K)$ such that for all $T \geq T_{c}$ and any $\omega(0) \in R\left(P_{N}\right)$,

$$
\frac{1}{T} \int_{0}^{T}\left\|P_{N} e^{t J L} \omega(0)\right\|_{X}^{2} d t \leq \kappa\|\omega(0)\|_{X}^{2} .
$$

In the next Lemma, we obtain the same estimates on the growth of solutions of (2.13), as in Lemma 2.3.

Lemma 2.8 Let $\omega(t)$ be a solution of (2.13) with $\omega(0) \in X_{1} \cap H^{1}(\mathbb{T})$. Then

$$
\|\omega(t)\|_{H^{1}} \leq C(1+t)\|\omega(0)\|_{H^{1}},
$$

for some constant $C$.

Proof. The proof is very similar to that of Lemma 2.3. We only sketch it briefly. By the conservation of $\langle L \omega, \omega\rangle$ for the equation (2.13) and the positivity of $\left.L\right|_{X_{1}}$, the $L^{2}$ norm of $\omega(t)$ is bounded by $\omega(0)$. Since $P_{1}$ is the projector of $X$ to $\operatorname{ker} L=\operatorname{ker}\left(1+\Delta^{-1}\right)$ and $\nabla$ is commutable with $1+\Delta^{-1}$, so $P_{1}$ is also commutable with $\nabla$. Then the estimates of $\partial \omega(t)$ follows in the same way as in the proof of Lemma 2.3.

Similarly, we can estimate the difference of solutions of (2.13) and (2.11).

Lemma 2.9 Let $\omega^{\nu}, \omega^{0}$ be the solutions of the projected linearized NS equation (1.3) and Euler equation (2.4) with the initial data $\omega^{\nu}(0) \in X_{1}$ and $\omega_{0} \in X_{1} \cap H^{1}$. Then there exists constants $C_{0}>0$ such that

$$
\frac{d}{d t}\left\|\omega^{\nu}-\omega^{0}\right\|_{X}^{2} \leq C_{0} \nu\left(1+t^{2}\right)\left\|\omega^{0}(t)\right\|_{H^{1}}^{2}
$$

for $t \in(0,+\infty)$.

The proof is the same as that of Lemma 2.6 by using the fact that $\left.L\right|_{X_{1}}>0$ and $\left\langle L\left(I-P_{1}\right) \cdot, \cdot\right\rangle=\langle L \cdot, \cdot\rangle$.

Then by the same proof of Theorem $1.1 \mathrm{i}$ ), we can show the enhanced damping for the solution $\omega_{1}(t)$ of the projected equation (2.11). More precisely, for any $\tau>0$ and $\delta>0$, if $\nu$ is small enough, then

$$
\left\|\omega_{1}\left(\frac{\tau}{\nu}\right)\right\|_{L^{2}}<\delta\left\|\omega_{1}(0)\right\|_{L^{2}} .
$$

Since $\omega_{1}(t)=\left(I-P_{1}\right) \omega(t)$, this proves Theorem $\left.1.1 \mathrm{ii}\right)$. 


\section{Metastability of nonlinear Navier-Stokes equa- tion}

In this section, we prove the metastability or enhanced damping for the nonlinear Navier-Stokes equation (1.1) on a torus $\mathbb{T}_{\alpha}(\alpha \geq 1)$. Let $P_{2}$ to be the projection of $L^{2}\left(\mathbb{T}_{\alpha}\right)$ to the subspace $W_{2}=\operatorname{span}\{\cos y, \sin y\}$. For any initial data $\omega(0) \in L^{2}$, let

$$
P_{2} \omega(0)=d_{1} \cos y+d_{2} \sin y=D \sin \left(y+y_{1}\right),
$$

where $D=\sqrt{d_{1}^{2}+d_{2}^{2}}$ and $y_{1}=\tan ^{-1}\left(d_{1} / d_{2}\right)$. When $\left\|\left(I-P_{2}\right) \omega(0)\right\|_{L^{2}}$ is small, we can equivalently consider the perturbation near the shear flow $U(y)=D \sin \left(y+y_{1}\right)$ with initial data $\omega(0)$ satisfying $P_{2} \omega(0)=0$, for which the analysis is almost the same as for the shear flow $U(y)=\sin y$. For simplicity, in the proof of Theorem 1.2, we only consider the perturbations near $U(y)=\sin y$ with $P_{2} \omega(0)=0$. As in the proof of Theorem 1.1 for the linearized NS equation, we will treat the high and low frequency parts of the non-shear perturbation separately. In particular, for the low frequency part, we will compare the solutions of the nonlinear NS equation and the linearized Euler equation, and then use the RAGE Theorem to control the time average. However, a significant difference with the linearized NS equation is that the shear and non-shear parts are strongly coupled for the nonlinear NS equation. Therefore, the main issue is to control the interaction terms. We will consider the equations on rectangular and square tori separately. On the square torus, the existence of anomalous modes makes the interactions terms considerably more subtle to handle.

\subsection{The case of rectangular torus}

Consider the nonlinear Navier-Stokes equation near the Kolmogorov flow

$$
\begin{aligned}
\partial_{t} \omega^{\nu} & =\nu \Delta \omega^{\nu}-e^{-\nu t}\left[\sin y \partial_{x}\left(1+\Delta^{-1}\right)\right] \omega^{\nu}+U^{\nu} \cdot \nabla \omega^{\nu} \\
& =L(t) \omega^{\nu}+U^{\nu} \cdot \nabla \omega^{\nu}
\end{aligned}
$$

on $\mathbb{T}_{\alpha}(\alpha>1)$, where $\omega^{\nu}, U^{\nu}$ are the perturbations of vorticity and velocity. We split $\omega^{\nu}, U^{\nu}$ into shear and non-shear components. More precisely, we write $\omega^{\nu}=\omega_{s}^{\nu}+\omega_{n}^{\nu}$, where the shear part

$$
\omega_{s}^{\nu}(t, y)=\frac{\alpha}{2 \pi} \int_{0}^{\frac{2 \pi}{\alpha}} \omega^{\nu}(t, x, y) d x=P_{0} \omega^{\nu},
$$


and non-shear part

$$
\omega_{n}^{\nu}(t, x, y)=\left(I-P_{0}\right) \omega^{\nu}=P_{\neq 0} \omega^{\nu} \in X .
$$

Correspondingly, $U^{\nu}=U_{s}^{\nu}+U_{n}^{\nu}$ and $\psi^{\nu}=\psi_{s}^{\nu}+\psi_{n}^{\nu}$ where $\psi^{\nu}$ is the stream function. We also denote

$$
U^{\nu}=\left(u^{\nu}, v^{\nu}\right), \quad U_{s}^{\nu}=\left(u_{s}^{\nu}(t, y), 0\right), U_{n}^{\nu}=\left(u_{n}^{\nu}, v_{n}^{\nu}\right) .
$$

Then the equation (3.1) can be written as

$$
\partial_{t} \omega_{s}^{\nu}=\nu \partial_{y y} \omega_{s}^{\nu}+P_{0}\left(U_{n}^{\nu} \cdot \nabla \omega_{n}^{\nu}\right)=\nu \partial_{y y} \omega_{s}^{\nu}+\partial_{y} P_{0}\left(v_{n}^{\nu} \omega_{n}^{\nu}\right)
$$

and

$$
\begin{aligned}
\partial_{t} \omega_{n}^{\nu} & =L(t) \omega_{n}^{\nu}+u_{s}^{\nu} \partial_{x} \omega_{n}^{\nu}+v_{n}^{\nu} \partial_{y} \omega_{s}^{\nu}+P_{\neq 0}\left(U_{n}^{\nu} \cdot \nabla \omega_{n}^{\nu}\right) \\
& =L(t) \omega_{n}^{\nu}+u_{s}^{\nu} \partial_{x} \omega_{n}^{\nu}+v_{n}^{\nu} \partial_{y} \omega_{s}^{\nu}+\partial_{x}\left(u_{n}^{\nu} \omega_{n}^{\nu}\right)+\partial_{y} P_{\neq 0}\left(v_{n}^{\nu} \omega_{n}^{\nu}\right) .
\end{aligned}
$$

First, we show that the dissipation law (1.11) also holds true for solutions of the nonlinear equation (3.1).

Lemma 3.1 Let $\omega^{\nu}(t)$ be a solution of (3.1) with the initial data $\omega^{\nu}(t) \in$ $L^{2}\left(\mathbb{T}_{\alpha}\right)$. Then

$$
\frac{d}{d t} \int_{\mathbb{T}_{\alpha}}\left(\left|\omega^{\nu}\right|^{2}-\left|\nabla \psi^{\nu}\right|^{2}\right) d x d y=-2 \nu \int_{\mathbb{T}_{\alpha}}\left(\left|\nabla \omega^{\nu}\right|^{2}-\left|\omega^{\nu}\right|^{2}\right) d x d y,
$$

for any $t>0$.

Proof. We have

$$
\begin{aligned}
\frac{d}{d t} \int_{\mathbb{T}_{\alpha}}\left(\left|\omega^{\nu}\right|^{2}-\left|\nabla \psi^{\nu}\right|^{2}\right) d x d y & =\int_{\mathbb{T}_{\alpha}} \omega_{t}^{\nu}\left(\omega^{\nu}-\psi^{\nu}\right) d x d y \\
& =\left\langle L(t) \omega^{\nu},\left(\omega^{\nu}-\psi^{\nu}\right)\right\rangle+\int_{\mathbb{T}_{\alpha}} U^{\nu} \cdot \nabla \omega^{\nu}\left(\omega^{\nu}-\psi^{\nu}\right) d x d y \\
& =-2 \nu \int_{\mathbb{T}_{\alpha}}\left(\left|\nabla \omega^{\nu}\right|^{2}-\left|\omega^{\nu}\right|^{2}\right) d x d y+\frac{1}{2} \int_{\mathbb{T}_{\alpha}} U^{\nu} \cdot \nabla \frac{1}{2} \omega^{\nu 2} d x d y \\
& \quad-\int_{\mathbb{T}_{\alpha}}\left(U^{\nu} \cdot \nabla \psi^{\nu}\right) \omega^{\nu} d x d y \\
& =-2 \nu \int_{\mathbb{T}_{\alpha}}\left(\left|\nabla \omega^{\nu}\right|^{2}-\left|\omega^{\nu}\right|^{2}\right) d x d y .
\end{aligned}
$$


In the above, we use the fact that $U^{\nu} \cdot \nabla \psi^{\nu}=0$ and

$$
\left\langle L(t) \omega^{\nu},\left(\omega^{\nu}-\psi^{\nu}\right)\right\rangle=-2 \nu \int_{\mathbb{T}_{\alpha}}\left(\left|\nabla \omega^{\nu}\right|^{2}-\left|\omega^{\nu}\right|^{2}\right) d x d y
$$

as in the proof of Lemma 2.1.

Denote $Y$ to be the space of mean zero functions in $L^{2}\left(\mathbb{T}_{\alpha}\right)$, depending only on $y$. Denote $Y_{1}=\left(I-P_{2}\right) Y$. Then the operator $L=1+\Delta^{-1}$ is positive on $Y_{1}$. There exists $c_{0}>0$ such that

$$
\int_{\mathbb{T}_{\alpha}}\left(\left|\omega^{\nu}\right|^{2}-\left|\partial_{y} \psi^{\nu}\right|^{2}\right) d x d y \geq c_{0}\left\|\omega^{\nu}\right\|_{L^{2}}^{2},
$$

and

$$
\int_{\mathbb{T}_{\alpha}}\left(\left|\partial_{y} \omega^{\nu}\right|^{2}-\left|\omega^{\nu}\right|^{2}\right) d x d y \geq c_{0}\|\omega\|_{H^{1}}^{2}
$$

for all $\omega \in Y_{1}$.

For a solution $\omega^{\nu}=\omega_{s}^{\nu}+\omega_{n}^{\nu}$ of the nonlinear NS equation (3.1), let $\omega_{s}^{\nu}=\omega_{s 1}^{\nu}+\omega_{s 2}^{\nu}$, where $\omega_{s 1}^{\nu}=P_{2} \omega_{s}^{\nu}$ and $\omega_{s 2}^{\nu} \in Y_{1}$. Then (3.4) implies that

$$
\begin{aligned}
& \frac{d}{d t}\left(\int_{\mathbb{T}_{\alpha}}\left(\left|\omega_{s 2}^{\nu}\right|^{2}-\left|\partial_{y} \psi_{s 2}^{\nu}\right|^{2}\right) d x d y+\left\|\omega_{n}^{\nu}\right\|_{X}^{2}\right) \\
& =-2 \nu\left(\int_{\mathbb{T}_{\alpha}}\left(\left|\partial_{y} \omega_{s 2}^{\nu}\right|^{2}-\left|\omega_{s 2}^{\nu}\right|^{2}\right) d x d y+\left\|\omega_{n}^{\nu}\right\|_{X^{1}}^{2}\right) .
\end{aligned}
$$

In particular, by the positivity estimates (2.2), (2.3), it follows from above that there exists $C>0$ such that

$$
\left\|\omega_{s 2}^{\nu}\right\|_{L^{2}}(t)+\left\|\omega_{n}^{\nu}\right\|_{L^{2}}(t) \leq C\left(\left\|\omega_{s 2}^{\nu}\right\|_{L^{2}}(0)+\left\|\omega_{n}^{\nu}\right\|(0)_{L^{2}}\right) \leq C d \nu,
$$

where we assume $\left\|\omega^{\nu}\right\|_{L^{2}}(0) \leq d \nu$ for a constant $d>0$ to be determined later. To estimate $\omega_{s 1}^{\nu}=a_{1} \cos y+a_{2} \sin y$, we project (3.2) to $\{\cos y, \sin y\}$ to get

$$
\begin{aligned}
& \frac{d}{d t} a_{1}=-\nu a_{1}-\int_{\mathbb{T}_{\alpha}} v_{n}^{\nu} \omega_{n}^{\nu} \sin y d x d y \\
& \frac{d}{d t} a_{2}=-\nu a_{2}+\int_{\mathbb{T}_{\alpha}} v_{n}^{\nu} \omega_{n}^{\nu} \cos y d x d y .
\end{aligned}
$$

Let

$$
a(t)=\left\|\omega_{s 1}^{\nu}\right\|_{L^{2}}=\sqrt{a_{1}^{2}+a_{2}^{2}},
$$


then by (3.9), we have

$$
\begin{aligned}
\frac{d a}{d t} & \leq-\nu a(t)+\sqrt{\left|\int_{\mathbb{T}_{\alpha}} v_{n}^{\nu} \omega_{n}^{\nu} \sin y d x d y\right|^{2}+\left|\int_{\mathbb{T}_{\alpha}} v_{n}^{\nu} \omega_{n}^{\nu} \cos y d x d y\right|^{2}} \\
& \leq-\nu a(t)+\left\|v_{n}^{\nu}\right\|(t)_{L^{2}}\left\|\omega_{n}^{\nu}\right\|(t)_{L^{2}} \\
& \leq-\nu a(t)+(C d \nu)^{2} .
\end{aligned}
$$

Therefore

$$
\left\|\omega_{s 1}^{\nu}\right\|_{L^{2}}=a(t) \leq e^{-\nu t} a(0)+(C d \nu)^{2} \int_{0}^{t} e^{-\nu(t-s)} d s \leq C d \nu .
$$

Combined with (3.8), it follows from above that

$$
\left\|\omega_{s}^{\nu}\right\|_{L^{2}}(t) \leq C d \nu, \text { for all } t>0 .
$$

In the dissipation law (3.7), $\omega_{s 2}^{\nu}$ and $\omega_{n}^{\nu}$ are coupled. In the next lemma, we show that when $d$ is small, the dissipation law for $\omega_{n}^{\nu}$ can be "separated" from (3.7).

Lemma 3.2 There exists a constant d depending only on $\alpha$, such that when $\left\|\omega^{\nu}\right\|_{L^{2}}(0) \leq d \nu$, then

$$
\frac{d}{d t} \int_{\mathbb{T}_{\alpha}}\left\|\omega_{n}^{\nu}\right\|_{X}^{2} \leq-\nu\left\|\omega_{n}^{\nu}\right\|_{X^{1}}^{2}
$$

Proof. By (3.2), we have

$$
\begin{aligned}
& \frac{d}{d t} \int_{\mathbb{T}_{\alpha}}\left(\left|\omega_{s 2}^{\nu}\right|^{2}-\left|\partial_{y} \psi_{s 2}^{\nu}\right|^{2}\right) d x d y \\
= & 2 \int_{\mathbb{T}_{\alpha}} \partial_{t} \omega_{s 2}^{\nu}\left(\omega_{s 2}^{\nu}-\psi_{s 2}^{\nu}\right) d x d y=2 \int_{\mathbb{T}_{\alpha}} \partial_{t} \omega_{s}^{\nu}\left(\omega_{s 2}^{\nu}-\psi_{s 2}^{\nu}\right) d x d y \\
= & -2 \nu \int_{\mathbb{T}_{\alpha}}\left(\left|\partial_{y} \omega_{s 2}^{\nu}\right|^{2}-\left|\omega_{s 2}^{\nu}\right|^{2}\right) d x d y+2 \int_{\mathbb{T}_{\alpha}} \partial_{y}\left(v_{n}^{\nu} \omega_{n}^{\nu}\right)\left(\omega_{s 2}^{\nu}-\psi_{s 2}^{\nu}\right) d x d y \\
\leq & -2 \nu \int_{\mathbb{T}_{\alpha}}\left(\left|\partial_{y} \omega_{s 2}^{\nu}\right|^{2}-\left|\omega_{s 2}^{\nu}\right|^{2}\right) d x d y+C\left\|\omega_{n}^{\nu}\right\|_{X^{1}}^{2}\left\|\omega_{s 2}^{\nu}\right\|_{L^{2}} \\
\leq & -2 \nu \int_{\mathbb{T}_{\alpha}}\left(\left|\partial_{y} \omega_{s 2}^{\nu}\right|^{2}-\left|\omega_{s 2}^{\nu}\right|^{2}\right) d x d y+C d \nu\left\|\omega_{n}^{\nu}\right\|_{X^{1}}^{2} .
\end{aligned}
$$


In the first inequality above, we use the Sobolev embedding and (2.3). Combined with (3.7), this gives

$$
\frac{d}{d t} \int_{\mathbb{T}_{\alpha}}\left\|\omega_{n}^{\nu}\right\|_{X}^{2} \leq-\nu\left\|\omega_{n}^{\nu}\right\|_{X^{1}}^{2}(2-C d) \leq-\nu\left\|\omega_{n}^{\nu}\right\|_{X^{1}}^{2},
$$

when $d \leq 1 / C$.

To use the RAGE theorem to control the low frequency part of $\omega_{n}^{\nu}$, we need to estimate the difference of the solutions of the nonlinear NS equation (3.1) and the linearized Euler equation (2.4).

Lemma 3.3 There exists $d>0$ such that for any solution $\omega^{\nu}$ of NavierStokes equation (3.1) with initial data satisfying $\left\|\omega^{\nu}\right\|_{L^{2}}(0) \leq d \nu$, and any solution $\omega^{0}$ of the linearized Euler equation (2.4) with initial data in $X \cap H^{1}$, we have

$$
\frac{d}{d t}\left\|\omega_{n}^{\nu}-\omega^{0}\right\|_{X}^{2} \leq C_{0} \nu\left(1+t^{2}\right)\left\|\omega^{0}(t)\right\|_{H^{1}}^{2}, \forall t>0,
$$

for some constant $C_{0}>0$. Here, $\omega_{n}^{\nu}=P_{\neq 0} \omega^{\nu}$ is the non-shear part of $\omega^{\nu}$.

Proof. Let $\psi_{n}^{\nu}, \psi^{0}$ be the corresponding stream functions. Denote $\omega=$ $\omega_{n}^{\nu}-\omega^{0}$ and $\psi=\psi_{n}^{\nu}-\psi^{0}$, then

$$
\begin{gathered}
\omega_{t}=-e^{-\nu t} \sin y \partial_{x}(\omega-\psi)-\left(e^{-\nu t}-1\right) \sin y \partial_{x}\left(\omega^{0}-\psi^{0}\right)+\nu \triangle \omega_{n}^{\nu} \\
+u_{s}^{\nu} \partial_{x} \omega_{n}^{\nu}+v_{n}^{\nu} \partial_{y} \omega_{s}^{\nu}+P_{\neq 0}\left(U_{n}^{\nu} \cdot \nabla \omega_{n}^{\nu}\right) .
\end{gathered}
$$

Thus

$$
\begin{gathered}
\frac{d}{d t} \frac{1}{2}\left\|\omega^{\nu}-\omega^{0}\right\|_{X}^{2}=\int_{\mathbb{T}_{\alpha}} \omega_{t}(\omega-\psi) d x d y \\
=\left[-\int_{\mathbb{T}_{\alpha}}\left(e^{-\nu t}-1\right) \sin y \partial_{x}\left(\omega^{0}-\psi^{0}\right)(\omega-\psi) d x d y+\nu \int_{\mathbb{T}_{\alpha}} \triangle \omega_{n}^{\nu}(\omega-\psi) d x d y\right] \\
+\int_{\mathbb{T}_{\alpha}} u_{s}^{\nu} \partial_{x} \omega_{n}^{\nu}(\omega-\psi) d x d y+\int_{\mathbb{T}_{\alpha}} v_{n}^{\nu} \partial_{y} \omega_{s}^{\nu}(\omega-\psi) d x d y+\int_{\mathbb{T}_{\alpha}} U_{n}^{\nu} \cdot \nabla \omega_{n}^{\nu}(\omega-\psi) d x d y \\
=I+I I+I I I+I V .
\end{gathered}
$$

Similar to the proof of Lemma 2.6, the first term can be estimated by

$$
I \leq \nu\left(-c_{0}\left\|\omega_{n}^{\nu}\right\|_{H^{1}}^{2}+C(1+t)\left\|\omega^{\nu}\right\|_{H^{1}}\left\|\omega^{0}\right\|_{H^{1}}\right) .
$$


For the last term, noticing that as in the proof of Lemma 3.1

$$
\int_{\mathbb{T}_{\alpha}} U_{n}^{\nu} \cdot \nabla \omega_{n}^{\nu}\left(\omega_{n}^{\nu}-\psi_{n}^{\nu}\right) d x d y=0,
$$

we have

$$
\begin{aligned}
I V & =-\int_{\mathbb{T}_{\alpha}} U_{n}^{\nu} \cdot \nabla \omega_{n}^{\nu}\left(\omega^{0}-\psi^{0}\right) d x d y=\int_{\mathbb{T}_{\alpha}} U_{n}^{\nu} \cdot \nabla\left(\omega^{0}-\psi^{0}\right) \omega_{n}^{\nu} d x d y \\
& \leq C\left\|U_{n}^{\nu}\right\|_{L^{4}}\left\|\omega^{0}\right\|_{H^{1}}\left\|\omega_{n}^{\nu}\right\|_{L^{4}} \leq C\left\|\omega_{n}^{\nu}\right\|_{L^{2}}\left\|\omega^{0}\right\|_{H^{1}}\left\|\omega_{n}^{\nu}\right\|_{H^{1}} \\
& \leq C d \nu\left\|\omega^{0}\right\|_{H^{1}}\left\|\omega_{n}^{\nu}\right\|_{H^{1}} .
\end{aligned}
$$

The second term is estimated by

$$
\begin{aligned}
I I & =\int_{\mathbb{T}_{\alpha}} u_{s}^{\nu} \partial_{x} \omega_{n}^{\nu}\left(\psi_{n}^{\nu}-\omega^{0}+\psi^{0}\right) d x d y \\
& =-\int_{\mathbb{T}_{\alpha}} u_{s}^{\nu} \omega_{n}^{\nu}\left(\partial_{x} \psi_{n}^{\nu}-\partial_{x}\left(\omega^{0}-\psi^{0}\right)\right) d x d y \\
& \leq\left\|u_{s}^{\nu}\right\|_{L^{\infty}}\left\|\omega_{n}^{\nu}\right\|_{L^{2}}\left\|v_{n}^{\nu}\right\|_{L^{2}}+\left\|u_{s}^{\nu}\right\|_{L^{\infty}}\left\|\omega_{n}^{\nu}\right\|_{L^{2}}\left\|\omega^{0}\right\|_{H^{1}} \\
& \leq C\left(\left\|\omega_{s}^{\nu}\right\|_{L^{2}}\left\|\omega_{n}^{\nu}\right\|_{H^{1}}^{2}+\left\|\omega_{s}^{\nu}\right\|_{L^{2}}\left\|\omega_{n}^{\nu}\right\|_{H^{1}}\left\|\omega^{0}\right\|_{H^{1}}\right) \\
& \leq C d \nu\left(\left\|\omega_{n}^{\nu}\right\|_{H^{1}}^{2}+\left\|\omega_{n}^{\nu}\right\|_{H^{1}}\left\|\omega^{0}\right\|_{H^{1}}\right),
\end{aligned}
$$

where we use (3.10) in the last inequality. Similarly,

$$
\begin{aligned}
I I I & =-\int_{\mathbb{T}_{\alpha}} U_{n}^{\nu} \cdot \nabla(\omega-\psi) \omega_{s}^{\nu} d x d y \\
& \leq C\left\|U_{n}^{\nu}\right\|_{L^{\infty}}\left(\left\|\omega_{n}^{\nu}\right\|_{H^{1}}+\left\|\omega^{0}\right\|_{H^{1}}\right)\left\|\omega_{s}^{\nu}\right\|_{L^{2}} \\
& \leq C d \nu\left(\left\|\omega_{n}^{\nu}\right\|_{H^{1}}^{2}+\left\|\omega^{0}\right\|_{H^{1}}\left\|\omega_{n}^{\nu}\right\|_{H^{1}}\right),
\end{aligned}
$$

where the embedding $\left\|U_{n}^{\nu}\right\|_{L^{\infty}} \leq C\left\|\omega_{n}^{\nu}\right\|_{H^{1}}$ and the bound (3.10) are used in the last inequality above. Combing above estimates, we get

$$
\begin{aligned}
\frac{d}{d t} \frac{1}{2}\left\|\omega^{\nu}-\omega^{0}\right\|_{X}^{2} & \leq \nu\left(-\left(c_{0}-C d\right)\left\|\omega_{n}^{\nu}\right\|_{H^{1}}^{2}+C(1+t)\left\|\omega_{n}^{\nu}\right\|_{H^{1}}\left\|\omega^{0}\right\|_{H^{1}}\right) \\
& \leq \nu\left(-\frac{1}{2} c_{0}\left\|\omega_{n}^{\nu}\right\|_{H^{1}}^{2}+C(1+t)\left\|\omega_{n}^{\nu}\right\|_{H^{1}}\left\|\omega^{0}\right\|_{H^{1}}\right) \\
& \leq C \nu\left(1+t^{2}\right)\left\|\omega^{0}(t)\right\|_{H^{1}}^{2},
\end{aligned}
$$

by choosing $d \leq \frac{1}{2 C}$.

By using Lemmas 3.1 and 3.3, Theorem 1.2 i) follows by the same arguments as in the proof of Theorem 1.1. 


\subsection{The case of square torus}

Consider the nonlinear Navier-Stokes equation (3.1) on the square torus $\mathbb{T}$, for initial data satisfying $P_{2} \omega(0)=0$. Compared with the rectangular torus, the new difficulty is the existence of anomalous modes $\{\cos x, \sin x\}$ in the kernel of $L=1+\Delta^{-1}$. We decompose the vorticity perturbation as

$$
\omega^{\nu}=\omega_{s}^{\nu}+\omega_{n}^{\nu}=\omega_{s 1}^{\nu}+\omega_{s 2}^{\nu}+\omega_{n 1}^{\nu}+\omega_{n 2}^{\nu},
$$

where the shear part $\omega_{s}^{\nu}$ is decomposed as in the rectangular case with $\omega_{s 1}^{\nu}=P_{2} \omega_{s}^{\nu}$ and $\omega_{s 2}^{\nu} \in Y_{1}$, and the non-shear part $\omega_{n}^{\nu}$ is decomposed as $\omega_{n 1}^{\nu}+\omega_{n 2}^{\nu}$ with $\omega_{n 1}^{\nu}=P_{1} \omega_{n}^{\nu}$ and $\omega_{n 2}^{\nu} \in X_{1}$. Here, we recall that $P_{1}$ is the projection to the anomalous space $W_{1}$ spanned by $\{\cos x, \sin x\}$ and $X_{1}$ is the orthogonal complement of $W_{1}$ in $X$. Correspondingly, the velocity $U^{\nu}$ and stream function $\psi$ are decomposed into four parts. Then the nonlinear term can be written as

$$
\begin{gathered}
U^{\nu} \cdot \nabla \omega^{\nu}=\left(U_{s 1}^{\nu}+U_{n 1}^{\nu}\right) \cdot \nabla\left(\omega_{n 2}^{\nu}-\psi_{n 2}^{\nu}\right)+U_{n 1}^{\nu} \cdot \nabla\left(\omega_{s 2}^{\nu}-\psi_{s 2}^{\nu}\right) \\
+U_{s 2}^{\nu} \cdot \nabla \omega_{n 2}^{\nu}+U_{n 2}^{\nu} \cdot \nabla \omega_{s 2}^{\nu}+U_{n 2}^{\nu} \cdot \nabla \omega_{n 2}^{\nu},
\end{gathered}
$$

where we use the observation

$$
U_{s 1}^{\nu} \cdot \nabla \omega_{n}^{\nu}+U_{n}^{\nu} \cdot \nabla \omega_{s 2}^{\nu}=U_{s 1}^{\nu} \cdot \nabla\left(\omega_{n}^{\nu}-\psi_{n}^{\nu}\right)=U_{s 1}^{\nu} \cdot \nabla\left(\omega_{n 2}^{\nu}-\psi_{n 2}^{\nu}\right)
$$

and similarly for other terms. The dissipation law (3.7) becomes

$$
\begin{aligned}
& \frac{d}{d t}\left(\int_{\mathbb{T}}\left(\left|\omega_{s 2}^{\nu}\right|^{2}-\left|\partial_{y} \psi_{s 2}^{\nu}\right|^{2}\right) d x d y+\left\|\omega_{n 2}^{\nu}\right\|_{X}^{2}\right) \\
& =-2 \nu\left(\int_{\mathbb{T}}\left(\left|\partial_{y} \omega_{s 2}^{\nu}\right|^{2}-\left|\omega_{s 2}^{\nu}\right|^{2}\right) d x d y+\left\|\omega_{n 2}^{\nu}\right\|_{X^{1}}^{2}\right) .
\end{aligned}
$$

By using the positivity of the above functional on $Y_{1}$ and $X_{1}$, this implies that

$$
\left\|\omega_{s 2}^{\nu}\right\|_{L^{2}}(t)+\left\|\omega_{n 2}^{\nu}\right\|_{L^{2}} \leq C\left(\left\|\omega_{s 2}^{\nu}\right\|_{L^{2}}(0)+\left\|\omega_{n 2}^{\nu}\right\|(0)_{L^{2}}\right) \leq C d \nu .
$$

We estimate $\left\|\omega_{s 1}^{\nu}\right\|_{L^{2}}(t)$ and $\left\|\omega_{n 1}^{\nu}\right\|_{L^{2}}$ below. Denote

$$
\omega_{s 1}^{\nu}=a_{1} \cos y+a_{2} \sin y, \omega_{n 1}^{\nu}=b_{1} \cos x+b_{2} \sin x,
$$


and

$$
a(t)=\left\|\omega_{s 1}^{\nu}\right\|_{L^{2}}(t)=\sqrt{a_{1}^{2}+a_{2}^{2}}, b(t)=\left\|\omega_{n 1}^{\nu}\right\|_{L^{2}}(t)=\sqrt{b_{1}^{2}+b_{2}^{2}} .
$$

Since $P_{2} \omega(0)=0$, we have $a(0)=0$. By (3.12), we have

$$
\begin{aligned}
\partial_{t} \omega_{s}^{\nu} & =\nu \partial_{y y} \omega_{s}^{\nu}+P_{0}\left(U_{n}^{\nu} \cdot \nabla \omega_{n}^{\nu}\right) \\
& =\nu \partial_{y y} \omega_{s}^{\nu}+P_{0}\left(U_{n 1}^{\nu} \cdot \nabla\left(\omega_{n 2}^{\nu}-\psi_{n 2}^{\nu}\right)+U_{n 2}^{\nu} \cdot \nabla \omega_{n 2}^{\nu}\right) .
\end{aligned}
$$

Projecting above to $\{\cos y, \sin y\}$ and using (3.14), we get

$$
\frac{d a_{i}}{d t} \leq-\nu a_{i}(t)+C d \nu\left(b(t)+\left\|\omega_{n 2}^{\nu}\right\|_{L^{2}}\right), i=1,2,
$$

and thus

$$
\frac{d a}{d t} \leq-\nu a(t)+C d \nu\left(b(t)+\left\|\omega_{n 2}^{\nu}\right\|_{L^{2}}\right)
$$

Projecting (3.1) to $\{\cos x, \sin x\}$ and using (3.12) (3.14), we get

$$
\frac{d b_{i}}{d t} \leq-\nu b_{i}(t)+C d \nu\left((a(t)+b(t))+\left\|\omega_{n 2}^{\nu}\right\|_{L^{2}}\right), i=1,2,
$$

and

$$
\frac{d b}{d t} \leq-\nu b(t)+C d \nu\left((a(t)+b(t))+\left\|\omega_{n 2}^{\nu}\right\|_{L^{2}}\right) .
$$

Let $e(t)=a(t)+b(t)$, then the combination of (3.16) and (3.17) yields

$$
\frac{d e}{d t} \leq-\nu(1-C d) e(t)+C d \nu\left\|\omega_{n 2}^{\nu}\right\|_{L^{2}} \leq-\frac{1}{2} \nu e(t)+C d \nu\left\|\omega_{n 2}^{\nu}\right\|_{L^{2}},
$$

by choosing $C d \leq \frac{1}{2}$. Thus by (3.14), we have

$$
\begin{aligned}
\left\|\omega_{s 1}^{\nu}\right\|_{L^{2}}(t)+\left\|\omega_{n 1}^{\nu}\right\|_{L^{2}} & =e(t) \\
& \leq e^{-\frac{1}{2} \nu t} e(0)+C d \nu \int_{0}^{t} e^{-\frac{1}{2} \nu(t-s)}\left\|\omega_{n 2}^{\nu}\right\|_{L^{2}}(s) d s \leq C d \nu .
\end{aligned}
$$

In the following lemma, we separate the dissipation law for $\omega_{n 2}^{\nu}$ from (3.13).

Lemma 3.4 There exists a constant $d>0$, such that when $\left\|\omega^{\nu}\right\|_{L^{2}}(0) \leq d \nu$, then

$$
\frac{d}{d t}\left\|\omega_{n 2}^{\nu}\right\|_{X}^{2} \leq-\nu\left\|\omega_{n 2}^{\nu}\right\|_{X^{1}}\left(\left\|\omega_{n 2}^{\nu}\right\|_{X^{1}}-\left\|\omega_{n 1}^{\nu}\right\|_{L^{2}}\right)
$$


Proof. By (3.15), we have

$$
\begin{aligned}
& \frac{d}{d t} \int_{\mathbb{T}}\left(\left|\omega_{s 2}^{\nu}\right|^{2}-\left|\partial_{y} \psi_{s 2}^{\nu}\right|^{2}\right) d x d y \\
= & 2 \int_{\mathbb{T}} \partial_{t} \omega_{s 2}^{\nu}\left(\omega_{s 2}^{\nu}-\psi_{s 2}^{\nu}\right) d x d y=2 \int_{\mathbb{T}} \partial_{t} \omega_{s}^{\nu}\left(\omega_{s 2}^{\nu}-\psi_{s 2}^{\nu}\right) d x d y \\
= & -2 \nu \int_{\mathbb{T}}\left(\left|\partial_{y} \omega_{s 2}^{\nu}\right|^{2}-\left|\omega_{s 2}^{\nu}\right|^{2}\right) d x d y \\
& \quad+2 \int_{\mathbb{T}_{\alpha}}\left(U_{n 1}^{\nu} \cdot \nabla\left(\omega_{n 2}^{\nu}-\psi_{n 2}^{\nu}\right)+U_{n 2}^{\nu} \cdot \nabla \omega_{n 2}^{\nu}\right)\left(\omega_{s 2}^{\nu}-\psi_{s 2}^{\nu}\right) d x d y \\
\leq & -2 \nu \int_{\mathbb{T}_{\alpha}}\left(\left|\partial_{y} \omega_{s 2}^{\nu}\right|^{2}-\left|\omega_{s 2}^{\nu}\right|^{2}\right) d x d y+C\left(\left\|\omega_{n 1}^{\nu}\right\|_{L^{2}}\left\|\omega_{n 2}^{\nu}\right\|_{X^{1}}+\left\|\omega_{n 2}^{\nu}\right\|_{X^{1}}^{2}\right)\left\|\omega_{s 2}^{\nu}\right\|_{L^{2}} \\
\leq & -2 \nu \int_{\mathbb{T}_{\alpha}}\left(\left|\partial_{y} \omega_{s 2}^{\nu}\right|^{2}-\left|\omega_{s 2}^{\nu}\right|^{2}\right) d x d y+C d \nu\left(\left\|\omega_{n 1}^{\nu}\right\|_{L^{2}}\left\|\omega_{n 2}^{\nu}\right\|_{X^{1}}+\left\|\omega_{n 2}^{\nu}\right\|_{X^{1}}^{2}\right) .
\end{aligned}
$$

By choosing $d$ such that $C d \leq 1$, (3.20) follows from above and (3.13).

Compared to the dissipation law (3.11) for the rectangular torus, from (3.20) we cannot even infer that $\left\|\omega_{n 2}^{\nu}\right\|_{X}^{2}$ is decreasing due to the interaction of $\omega_{n 2}^{\nu}$ and $\omega_{n 1}^{\nu}$. The dissipation law (3.13) only implies that

$$
\begin{aligned}
& \int_{\mathbb{T}}\left(\left|\omega_{s 2}^{\nu}\right|^{2}(t)-\left|\partial_{y} \psi_{s 2}^{\nu}\right|^{2}(t)\right) d x d y+\left\|\omega_{n 2}^{\nu}(t)\right\|_{X}^{2} \\
& \leq \int_{\mathbb{T}}\left(\left|\omega_{s 2}^{\nu}\right|^{2}(0)-\left|\partial_{y} \psi_{s 2}^{\nu}\right|^{2}(0)\right) d x d y+\left\|\omega_{n 2}^{\nu}(0)\right\|_{X}^{2},
\end{aligned}
$$

while the shear term $\omega_{s 2}^{\nu}$ cannot be separated from above. Below, we show that $\left\|\omega_{n 2}^{\nu}(t)\right\|_{X}$ and $\left\|\omega_{n 1}^{\nu}\right\|_{L^{2}}$ are controlled by their initial values with a factor $e^{C \nu t}$, which is enough for proving the enhanced damping.

Lemma 3.5 There exists $d>0$, such that when $\left\|\omega^{\nu}\right\|_{L^{2}}(0) \leq d \nu$, then

$$
\left\|\omega_{n 2}^{\nu}(t)\right\|_{X}^{2}+\left\|\omega_{n 1}^{\nu}\right\|_{L^{2}}(t)^{2}+\left\|\omega_{s 1}^{\nu}\right\|_{L^{2}}^{2}(t) \leq e^{C_{1} \nu t}\left\|\omega_{n}^{\nu}(0)\right\|_{L^{2}}^{2},
$$

for some constant $C_{1}>0$.

Proof. It follows from (3.20) that

$$
\frac{d}{d t}\left\|\omega_{n 2}^{\nu}\right\|_{X}^{2} \leq \frac{1}{4} \nu\left\|\omega_{n 1}^{\nu}\right\|_{L^{2}}^{2}
$$


From (3.18), we have

$$
\frac{d e^{2}}{d t} \leq-\nu e(t)^{2}+C d \nu\left\|\omega_{n 2}^{\nu}\right\|_{X} e(t) \leq \frac{1}{4}(C d)^{2} \nu\left\|\omega_{n 2}^{\nu}\right\|_{X}^{2} .
$$

Combining above and denoting $C_{1}=\max \left\{\frac{1}{4}, \frac{1}{4}(C d)^{2}\right\}$, then we have

$$
\begin{aligned}
\frac{d}{d t}\left(\left\|\omega_{n 2}^{\nu}\right\|_{X}^{2}+e(t)^{2}\right) & \leq C_{1} \nu\left(\left\|\omega_{n 2}^{\nu}\right\|_{X}^{2}+\left\|\omega_{n 1}^{\nu}\right\|_{L^{2}}^{2}\right) \\
& \leq C_{1} \nu\left(\left\|\omega_{n 2}^{\nu}\right\|_{X}^{2}+e(t)^{2}\right) .
\end{aligned}
$$

Since $\left\|\omega_{s 1}^{\nu}\right\|_{L^{2}}(0)=0$, we get

$$
\begin{aligned}
\left\|\omega_{n 2}^{\nu}\right\|_{X}^{2}+e(t)^{2} & \leq e^{C_{1} \nu t}\left(\left\|\omega_{n 2}^{\nu}\right\|_{X}^{2}(0)+\left\|\omega_{n 1}^{\nu}\right\|_{L^{2}}^{2}(0)\right) \\
& \leq e^{C_{1} \nu t}\left(\left\|\omega_{n 2}^{\nu}\right\|_{L^{2}}^{2}(0)+\left\|\omega_{n 1}^{\nu}\right\|_{L^{2}}^{2}(0)\right)=e^{C_{1} \nu t}\left\|\omega_{n}^{\nu}(0)\right\|_{L^{2}}^{2} .
\end{aligned}
$$

This finishes the proof of the lemma.

Our next lemma is to estimate the difference of $\omega_{n 2}^{\nu}$ and the solution of the linearized Euler equation.

Lemma 3.6 There exists $d>0$ such that for any solution $\omega^{\nu}$ of the NavierStokes equation (3.3) on $\mathbb{T}$ with initial data satisfying $\left\|\omega^{\nu}\right\|_{L^{2}}(0) \leq d \nu$, and any solution $\omega^{0}$ of the linearized Euler equation (2.4) with initial data in $X_{1} \cap H^{1}$, we have

$$
\frac{d}{d t}\left\|\omega_{n 2}^{\nu}-\omega^{0}\right\|_{X}^{2} \leq C_{0} \nu\left(\left(1+t^{2}\right)\left\|\omega^{0}(t)\right\|_{H^{1}}^{2}+\left\|\omega_{n 1}^{\nu}\right\|_{L^{2}}^{2}+\left\|\omega_{n 1}^{\nu}\right\|_{L^{2}}\left\|\omega^{0}\right\|_{H^{1}}\right),
$$

$\forall t>0$, for some constant $C_{0}>0$. Here, $\omega_{n 2}^{\nu}=\left(I-P_{1}\right) P_{\neq 0} \omega^{\nu}$ is the nonshear part of $\omega^{\nu}$ with anomalous modes removed.

Proof. Denote $\omega=\omega_{n 2}^{\nu}-\omega^{0}$ and $\psi=\psi_{n 2}^{\nu}-\psi^{0}$, then

$$
\begin{gathered}
\omega_{t}=-e^{-\nu t}\left(I-P_{1}\right) \sin y \partial_{x}(\omega-\psi)-\left(e^{-\nu t}-1\right)\left(I-P_{1}\right) \sin y \partial_{x}\left(\omega^{0}-\psi^{0}\right)+\nu \triangle \omega_{n 2}^{\nu} \\
+\left(I-P_{1}\right) P_{\neq 0}\left(\left(U_{s 1}^{\nu}+U_{n 1}^{\nu}\right) \cdot \nabla\left(\omega_{n 2}^{\nu}-\psi_{n 2}^{\nu}\right)\right)+\left(I-P_{1}\right)\left(U_{n 1}^{\nu} \cdot \nabla\left(\omega_{s 2}^{\nu}-\psi_{s 2}^{\nu}\right)\right) \\
+\left(I-P_{1}\right)\left(U_{s 2}^{\nu} \cdot \nabla \omega_{n 2}^{\nu}+U_{n 2}^{\nu} \cdot \nabla \omega_{s 2}^{\nu}\right)+\left(I-P_{1}\right) P_{\neq 0}\left(U_{n 2}^{\nu} \cdot \nabla \omega_{n 2}^{\nu}\right) .
\end{gathered}
$$

So we have

$$
\frac{d}{d t} \frac{1}{2}\left\|\omega_{n 2}^{\nu}-\omega^{0}\right\|_{X}^{2}=\int_{\mathbb{T}_{\alpha}} \omega_{t}(\omega-\psi) d x d y
$$




$$
\begin{aligned}
=[- & \left.\int_{\mathbb{T}}\left(e^{-\nu t}-1\right) \sin y \partial_{x}\left(\omega^{0}-\psi^{0}\right)(\omega-\psi) d x d y+\nu \int_{\mathbb{T}_{\alpha}} \Delta \omega_{n 2}^{\nu}(\omega-\psi) d x d y\right] \\
& +\left[\int_{\mathbb{T}}\left(U_{s 2}^{\nu} \cdot \nabla \omega_{n 2}^{\nu}+U_{n 2}^{\nu} \cdot \nabla \omega_{s 2}^{\nu}\right)(\omega-\psi) d x d y+\int_{\mathbb{T}} U_{n 2}^{\nu} \cdot \nabla \omega_{n 2}^{\nu}(\omega-\psi) d x d y\right] \\
& -\left[\int_{\mathbb{T}}\left(U_{s 1}^{\nu}+U_{n 1}^{\nu}\right) \cdot \nabla\left(\omega_{n 2}^{\nu}-\psi_{n 2}^{\nu}\right)\left(\omega^{0}-\psi^{0}\right) d x d y\right] \\
& +\int_{\mathbb{T}} U_{n 1}^{\nu} \cdot \nabla\left(\omega_{s 2}^{\nu}-\psi_{s 2}^{\nu}\right)(\omega-\psi) d x d y \\
=I+ & I I+I I I+I V .
\end{aligned}
$$

For the first three terms, as in the proof of Lemma 3.3, we get

$$
I+I I+I I I \leq-\frac{1}{2} c_{0} \nu\left\|\omega_{n 2}^{\nu}\right\|_{H^{1}}^{2}+C \nu(1+t)\left\|\omega_{n 2}^{\nu}\right\|_{H^{1}}\left\|\omega^{0}\right\|_{H^{1}},
$$

by choosing $d>0$ small enough. The last term is estimated by

$$
\begin{aligned}
I V & =-\int_{\mathbb{T}} U_{n 1}^{\nu} \cdot \nabla\left[\left(\omega_{n 2}^{\nu}-\psi_{n 2}^{\nu}\right)-\left(\omega^{0}-\psi^{0}\right)\right]\left(\omega_{s 2}^{\nu}-\psi_{s 2}^{\nu}\right) d x d y \\
& \leq C \nu\left\|\omega_{n 1}^{\nu}\right\|_{L^{2}}\left(\left\|\omega_{n 2}^{\nu}\right\|_{H^{1}}+\left\|\omega^{0}\right\|_{H^{1}}\right) .
\end{aligned}
$$

Combining above, we have

$$
\frac{d}{d t}\left\|\omega_{n 2}^{\nu}-\omega^{0}\right\|_{X}^{2} \leq C_{0} \nu\left(\left(1+t^{2}\right)\left\|\omega^{0}\right\|_{H^{1}}^{2}+\left\|\omega_{n 1}^{\nu}\right\|_{L^{2}}^{2}+\left\|\omega_{n 1}^{\nu}\right\|_{L^{2}}\left\|\omega^{0}\right\|_{H^{1}}\right) .
$$

We are now ready to prove Theorem $1.2 \mathrm{ii})$.

Proof of Theorem 1.2 ii). For any fixed $\delta, \tau>0$, suppose that (1.9) is not true. Then

$$
\left\|\omega_{n 2}^{\nu}(t)\right\|_{L^{2}} \geq \delta\left\|\omega_{n}^{\nu}(0)\right\|_{L^{2}}, \quad \forall 0 \leq t \leq \frac{\tau}{\nu}
$$

where $\omega^{\nu}(t)$ is the solution of $(3.1)$ with initial data $\omega^{\nu}(0) \in L^{2}(\mathbb{T})$ satisfying $P_{2} \omega^{\nu}(0)=0$ and $\left\|\omega^{\nu}(0)\right\|_{L^{2}} \leq d \nu$. Here, $d$ is a constant chosen such that Lemmas 3.4, 3.5 and 3.6 hold true. Then by (3.21) and (3.23), for any $t, t^{\prime} \in\left(0, \frac{\tau}{\nu}\right)$, we have

$$
\begin{aligned}
\left\|\omega_{n 1}^{\nu}(t)\right\|_{L^{2}} & \leq e^{\frac{1}{2} C_{1} \tau}\left\|\omega_{n}^{\nu}(0)\right\|_{L^{2}}^{2} \leq \frac{1}{\delta} e^{\frac{1}{2} C_{1} \tau}\left\|\omega_{n 2}^{\nu}\left(t^{\prime}\right)\right\|_{L^{2}} \\
& \leq \frac{c_{0}}{\delta} e^{\frac{1}{2} C_{1} \tau}\left\|\omega_{n 2}^{\nu}\left(t^{\prime}\right)\right\|_{X} .
\end{aligned}
$$


Choose $\lambda_{N}$ to be big enough such that

$$
\exp \left(-\left(\frac{\lambda_{N}}{2}-\sqrt{\frac{\lambda_{N}}{2}} \frac{c_{0}}{\delta} e^{\frac{1}{2} C_{1} \tau}\right) \tau\right)<c_{0} \delta^{2},
$$

where $C_{1}, c_{0}$ are the constants in (3.21) and (2.12) respectively. Suppose that $\omega_{n 2}^{\nu}(t) \in R\left(I-P_{N}\right)$, that is,

$$
\left\|\omega_{n 2}^{\nu}(t)\right\|_{X^{1}}^{2}>\lambda_{N}\left\|\omega_{n 2}^{\nu}(t)\right\|_{X}^{2}
$$

is true for $t$ in some interval $(a, b) \subset(0, \tau / \nu)$. Then by (3.20) and (3.24), we have

$$
\left\|\omega^{\nu}(b)\right\|_{X}^{2} \leq \exp \left(-\nu \sqrt{\lambda_{N}}\left(\sqrt{\lambda_{N}}-\frac{c_{0}}{\delta} e^{\frac{1}{2} C_{1} \tau}\right)(b-a)\right)\left\|\omega^{\nu}(a)\right\|_{X}^{2} .
$$

Denote $t_{1}=T_{c}\left(N, \frac{1}{10},\right)$ to be such that the RAGE Lemma (2.14) is true for $\kappa=1 / 10$ and all $T \geq T_{c}$. For any $t_{0} \in(0, \tau / \nu)$ satisfying

$$
\left\|\omega_{n 2}^{\nu}\left(t_{0}\right)\right\|_{X^{1}}^{2} \leq \lambda_{N}\left\|\omega_{n 2}^{\nu}\left(t_{0}\right)\right\|_{X}^{2}
$$

let $\omega^{0}(t)\left(t \in\left[t_{0}, t_{0}+t_{1}\right]\right)$ be the solution of (2.4) with $\omega^{0}\left(t_{0}\right)=\omega_{n 2}^{\nu}\left(t_{0}\right)$. By (3.22), (3.24) and Lemma 3.5, we have

$$
\begin{aligned}
\left\|\omega_{n 2}^{\nu}\left(t_{0}+t\right)-\omega^{0}\left(t_{0}+t\right)\right\|_{X}^{2} \leq & C_{2} \nu\left\{\left(1+t^{5}\right)\left\|\omega_{n 2}^{\nu}\left(t_{0}\right)\right\|_{X^{1}}^{2}+\left(\frac{c_{0}}{\delta}\right)^{2} e^{C_{1} \tau}\left\|\omega_{n 2}^{\nu}\left(t_{0}\right)\right\|_{X}^{2}\right. \\
& \left.+\frac{c_{0}}{\delta} e^{\frac{1}{2} C_{1} \tau}\left(1+t^{2}\right)\left\|\omega_{n 2}^{\nu}\left(t_{0}\right)\right\|_{X}\left\|\omega_{n 2}^{\nu}\left(t_{0}\right)\right\|_{X^{1}}\right\}
\end{aligned}
$$

for any $t \in\left[0, t_{1}\right]$ and some constant $C_{2}>0$ independent of $\tau, \nu$. Let $\nu(\delta, \tau)$ be such that

$$
C_{2} \nu(\delta, \tau)\left\{\left(1+t_{1}^{5}\right) \lambda_{N}+\left(\frac{c_{0}}{\delta}\right)^{2} e^{C_{1} \tau}+\frac{c_{0}}{\delta} e^{\frac{1}{2} C_{1} \tau}\left(1+t_{1}^{2}\right) \sqrt{\lambda_{N}}\right\}<\frac{1}{10} .
$$

By (3.26), when $0<\nu<\nu(\delta, \tau)$, we have

$$
\left\|\omega_{n 2}^{\nu}(t)-\omega^{0}(t)\right\|_{X}^{2} \leq \frac{1}{10}\left\|\omega_{n 2}^{\nu}\left(t_{0}\right)\right\|_{X}^{2}, \forall t \in\left[t_{0}, t_{0}+t_{1}\right] .
$$

Then as in the proof of Theorem 1.1, by using the RAGE theorem for $\omega^{0}(t)$, we obtain

$$
\int_{t_{0}}^{t_{0}+t_{1}}\left\|\omega_{n 2}^{\nu}(t)\right\|_{X^{1}}^{2} d t \geq \frac{\lambda_{N} t_{1}}{2}\left\|\omega_{n 2}^{\nu}\left(t_{0}\right)\right\|_{X}^{2} .
$$


Thus by (3.20) and (3.24), we have

$$
\begin{aligned}
&\left\|\omega_{n 2}^{\nu}\left(t_{0}+t_{1}\right)\right\|_{X}^{2} \leq\left\|\omega_{n 2}^{\nu}\left(t_{0}\right)\right\|_{X}^{2}-\nu \int_{t_{0}}^{t_{0}+t_{1}}\left\|\omega_{n 2}^{\nu}\right\|_{X^{1}}\left(\left\|\omega_{n 2}^{\nu}\right\|_{X^{1}}-\left\|\omega_{n 1}^{\nu}\right\|_{L^{2}}\right) d t \\
& \leq\left\|\omega_{n 2}^{\nu}\left(t_{0}\right)\right\|_{X}^{2}-\nu \int_{t_{0}}^{t_{0}+t_{1}}\left\|\omega_{n 2}^{\nu}\right\|_{X^{1}}^{2} d t \\
& \quad+\nu\left(\int_{t_{0}}^{t_{0}+t_{1}}\left\|\omega_{n 2}^{\nu}\right\|_{X^{1}}^{2} d t\right)^{\frac{1}{2}} \sqrt{t_{1}} \frac{c_{0}}{\delta} e^{\frac{1}{2} C_{1} \tau}\left\|\omega_{n 2}^{\nu}\left(t_{0}\right)\right\|_{X} \\
& \leq\left\|\omega_{n 2}^{\nu}\left(t_{0}\right)\right\|_{X}^{2}\left(1-\frac{\lambda_{N}}{2}+\sqrt{\frac{\lambda_{N}}{2}} \frac{c_{0}}{\delta} e^{\frac{1}{2} C_{1} \tau}\right) \nu t_{1} . \\
& \leq\left\|\omega_{n 2}^{\nu}\left(t_{0}\right)\right\|_{X}^{2} \exp \left(-\left(\frac{\lambda_{N}}{2}-\sqrt{\frac{\lambda_{N}}{2}} \frac{c_{0}}{\delta} e^{\frac{1}{2} C_{1} \tau}\right) \nu t_{1}\right) .
\end{aligned}
$$

Splitting the interval $\left[0, \frac{\tau}{\nu}\right]$ into a union of intervals such that either (3.25) or (3.27) is true, then we have

$$
\begin{aligned}
\left\|\omega_{n 2}^{\nu}\left(\frac{\tau}{\nu}\right)\right\|_{X}^{2} & \leq \exp \left(-\left(\frac{\lambda_{N}}{2}-\sqrt{\frac{\lambda_{N}}{2}} \frac{c_{0}}{\delta} e^{\frac{1}{2} C_{1} \tau}\right) \tau\right)\left\|\omega_{n 2}^{\nu}(0)\right\|_{X}^{2} \\
& <c_{0} \delta^{2}\left\|\omega_{n 2}^{\nu}(0)\right\|_{X}^{2} .
\end{aligned}
$$

This implies that

$$
\left\|\omega_{n 2}^{\nu}\left(\frac{\tau}{\nu}\right)\right\|_{L^{2}}<\delta\left\|\omega_{n 2}^{\nu}(0)\right\|_{L^{2}} \leq \delta\left\|\omega_{n}^{\nu}(0)\right\|_{L^{2}},
$$

which is in contradiction to the assumption (3.23). This finishes the proof of Theorem 1.2 ii).

Remark 3.1 By (3.14) and (3.19), we have the following Liapunov stability result

$$
\|\omega(t)\|_{L^{2}} \leq C\left\|\left(I-P_{2}\right) \omega(0)\right\|_{L^{2}}, \forall t>0,
$$

for some constant $C>0$ and any solution $\omega(t)$ of the NS equation (3.1). Thus for initial data $\omega(0)$ satisfying

$$
\left\|\left(I-P_{2}\right) \omega(0)\right\|_{L^{2}} \leq \frac{1}{C} d \nu,
$$


we can repeatedly use Theorem 1.2 ii) to get the rapid decay of the nonshear part with anomalous modes removed, before the dissipation term takes over. The same remark applies to the rectangular torus case to get the rapid damping of the non-shear part.

Remark 3.2 In Theorem 1.2 ii), the non-shear part removing the anomalous modes is reduced to a factor $\delta$ of the initial norm of the whole nonshear part. This is different from the result (1.7) for the linearized NS equation on a square torus, where the anomalous modes can be separated. The nonlinear coupling due to the anomalous modes can be seen from the term $U_{n 1}^{\nu} \cdot \nabla\left(\omega_{s 2}^{\nu}-\psi_{s 2}^{\nu}\right)$ in (3.12), which reflects the nonlinear interaction of the anomalous modes and the shear part. For the rectangular torus, there is no such interaction term and the nonlinear enhanced damping result is consistent with that for the linearized NS equation.

\subsection{Further issues and dipole states}

We comment on some further issues. First, it would be very interesting to enlarge the metastability basin from $O(\nu)$ in Theorem 1.2 to be $O\left(\nu^{\alpha}\right)$ $(0<\alpha<1)$ or independent of $\nu$ if possible. Also, it is desirable to improve the decay time scale from $O(\tau / \nu)$ to $O(1 / \sqrt{\nu})$ as given in [3] for the approximated linearized equation (1.4). This might require us to work on initial data of higher regularity. We note that the time scale $O(1 / \sqrt{\nu})$ in [3] was obtained for initial data in $H^{1}$.

Numerical simulations ([6]) suggest that on the rectangular torus the bar states (i.e. Kolmogorov flows) are usually observed. However, on the square torus ([22]), the dipole states of the form $\omega_{0}=\cos x+\cos y$ or $\sin x+\sin y$ appear more often than the bar states. These dipole states are also maximum entropy solutions of the 2D Euler equation, and hence likely candidates for relevant quasi-stationary states by the statistical approaches of 2D turbulence (e.g. [19]). The dipole states represent nonparallel flows with saddle points on the stream lines and therefore are more difficult to study. Consider the dipole with $\omega_{0}=\cos x+\cos y$, then the quasi-stationary Navier-Stokes solution is $\omega^{\nu}(t, x, y)=e^{-\nu t}(\cos x+\cos y)$. The linearized NS equation around it becomes

$$
\partial_{t} \omega=\nu \Delta \omega+e^{-\nu t}\left[\left(\sin y \partial_{x}-\sin x \partial_{y}\right)\left(1+\Delta^{-1}\right)\right] \omega .
$$


There are some similarities with the linearized equation (1.3) near bar states. First, the same dissipation law (1.11) holds true for (3.30). The linearized Euler operator is of the Hamiltonian form

$$
\left(\sin y \partial_{x}-\sin x \partial_{y}\right)\left(1+\Delta^{-1}\right)=J L
$$

with

$$
J=\sin y \partial_{x}-\sin x \partial_{y}, \quad L=1+\Delta^{-1} .
$$

Consider the energy space $X$ to be the set of $L^{2}$ functions with zero mean. Define $P$ to be the projection of $L^{2}$ to ker $J$. It was shown in [11] that for any $\phi \in L^{2}$,

$$
\left.P \phi\right|_{\gamma_{i}(c)}=\frac{\oint_{\gamma_{i}(c)} \frac{\phi(x, y)}{\left|\nabla \psi_{0}\right|} d l}{\oint_{\gamma_{i}(c)} \frac{1}{\left|\nabla \psi_{0}\right|} d l},
$$

where $c$ is in the range of $\psi_{0}=\cos x+\cos y$ and $\gamma_{i}(c)$ is a branch of $\left\{\psi_{0}=c\right\}$. Define the operator $A, A_{0}: H^{2} \cap X \rightarrow X$ by

$$
A=-\Delta-1+P, \quad A_{0}=-\Delta-1
$$

We note that $A \geq A_{0} \geq 0$ and

$$
\operatorname{ker} A_{0}=\operatorname{ker} L=\{\cos x, \sin x, \cos y, \sin y\} .
$$

Therefore ker $A \subset \operatorname{ker} A_{0}$ and by Proposition 2.8 and Lemma 11.3 of [15], we have the decomposition

$$
L^{2}=\operatorname{ker}(J L)+R(J) .
$$

Here, $\operatorname{ker}(J L) \cap R(J) \subset \operatorname{ker} L$ and $\operatorname{ker}(J L), R(J)$ are both invariant under $J L$. The space ker $(J L)$ corresponds to the steady solution space of the linearized Euler equation $\partial_{t} \omega=J L \omega$. Different from the case of bar states where $\operatorname{ker}(J L)$ is the space of shear flows, for the dipole states the steady space ker $(J L)$ has a more complicated structure. We can restrict the Euler semigroup $e^{t J L}$ on the invariant subspace $R(J)$. Denote $P_{3}$ to be the orthogonal projection of $L^{2}(\mathbb{T})$ to

$$
\text { ker } L=\{\cos x, \sin x, \cos y, \sin y\} .
$$

We have the following RAGE type result for $J L$ on $R(J)$. 
Proposition 3.1 Suppose that the operator JL defined in (3.31) has no nonzero purely imaginary eigenvalues. Let $B$ be any compact operator in $L^{2}(\mathbb{T})$. Then for any $\omega(0) \in R(J)$, we have

$$
\frac{1}{T} \int_{0}^{T}\left\|B\left(I-P_{3}\right) e^{i t L} \omega(0)\right\|_{L^{2}}^{2} d t \rightarrow 0 \text {, when } T \rightarrow \infty .
$$

Proof. The proof is similar to Lemma 2.7 for bar states. We only sketch it. For any solution $\omega(t)$ of the equation $\partial_{t} \omega=J L \omega$ with $\omega(0) \in R(J)$, define $\omega_{1}(t)=\left(I-P_{3}\right) \omega(t)$ and let $X_{1}=\left(I-P_{3}\right) R(J)$. Then $\omega_{1}(t)$ satisfies the equation $\partial_{t} \omega_{1}=\left(I-P_{3}\right) J L \omega$ on the space $X_{1}$. Since $\left.L\right|_{X_{1}}>0$, the operator $\left(I-P_{3}\right) J L$ is anti-selfadjoint on $\left(X_{1},\langle L \cdot, \cdot\rangle\right)$. Our assumption on the spectrum of $J L$ implies that $\left(I-P_{3}\right) J L$ has no nonzero purely imaginary eigenvalues. To show that the operator $\left(I-P_{3}\right) J L$ has purely continuous spectrum on $X_{1}$, it remains to show that 0 is not an embedded eigenvalue of $\left(I-P_{3}\right) J L$. Suppose otherwise, there exists $0 \neq \omega \in X_{1}$ such that $\left(I-P_{3}\right) J L \omega=0$. Let $\psi_{1}=L \omega$, then $J \psi_{1}=P_{3} J L \omega \in \operatorname{ker} L$. Denote

$$
J \psi_{1}=a_{1} \cos x+a_{2} \cos y+b_{1} \sin x+b_{2} \sin y .
$$

Since $\cos y+\cos x \in \operatorname{ker} J \perp J \psi_{1}$, we have $a_{1}+a_{2}=0$. Let $a_{1}=-a_{2}=a$. It is easy to see that for any $c \neq 0$ in the range of $\psi_{0}=\cos x+\cos y$, each of the two branches of $\left\{\psi_{0}=c\right\}$ is symmetric to $x, y$ in the sense that both $(x, y)$ and $(y, x)$ are on the branch. Since any function $\phi$ in ker $J$ takes the form (3.32), we conclude that $\cos x-\cos y \perp$ ker $J$, which implies that $\cos x-\cos y \in R(J)$. So there exists a double periodic function $\psi_{2}$ such that $J \psi_{2}=\cos x-\cos y$. By noting that $J x=\sin y$ and $J y=-\sin x$, it follows from (3.35) that $J\left(\psi_{1}-a \psi_{2}+b_{1} y-b_{2} x\right)=0$. Then by (3.32), the function $\psi_{1}-a \psi_{2}+b_{1} y-b_{2} x$ must take constant on each branch of the level set $\left\{\psi_{0}=c\right\}$. Since $\psi_{0}$ is double periodic in $x, y$, this implies that $\psi_{1}-a \psi_{2}+b_{1} y-b_{2} x$ is also double periodic. This contradiction shows that 0 is not an embedded eigenvalue of $\left(I-P_{3}\right) J L \omega$. Then (3.34) follows from the standard RAGE theorem.

Even with the dissipation law (1.11) and above RAGE theorem, there are still significant differences with the bar states to get the linear enhanced damping for dipoles, besides the issue of proving the non-existence of imaginary eigenvalues of $J L$. The most important difference is that the decomposition (3.33) is no longer invariant when the viscosity is added on. It is under investigation to find a subspace of initial data such that the enhanced damping is true for dipoles. 


\section{Linear inviscid damping of shear flows}

Consider a shear flow $u_{0}=(U(y), 0)$ in a channel $\left\{y_{1} \leq y \leq y_{2}\right\}$ or on a torus. The linearized Euler equation can be written as

$$
\omega_{t}+U(y) \partial_{x} \omega+U^{\prime \prime}(y) \partial_{x} \psi=0
$$

where $\omega$ and $\psi=(-\Delta)^{-1} \omega$ are the vorticity and stream functions respectively.

\subsection{Stable case}

We consider two classes of stable shear flows.

Class 1: $U^{\prime \prime} \neq 0$, that is, $U$ has no inflection points. This case is restricted to a channel, since such flows can not exist on a torus. By the classical Rayleigh inflection point theorem, $(U(y), 0)$ is linearly stable. Suppose $U^{\prime \prime}>$ 0 , choose a constant $U_{s}<\min U$. Then in the frame $\left(x-U_{s} t, y, t\right)$, the equation (4.1) becomes

$$
\omega_{t}+\left(U(y)-U_{s}\right) \partial_{x} \omega+U^{\prime \prime}(y) \partial_{x} \psi=0
$$

Define

$$
K_{1}(y)=\frac{U^{\prime \prime}(y)}{U(y)-U_{s}}>0 .
$$

Let the $x$ period be $2 \pi / \alpha$ for any $\alpha>0$. Define the non-shear space on the periodic channel $S_{2 \pi / \alpha} \times\left[y_{1}, y_{2}\right]$ by

$$
X=\left\{\omega=\sum_{k \in \mathbf{Z}, k \neq 0} e^{i k \alpha x} \omega_{k}(y),\|\omega\|_{X}^{2}=\left\|\frac{1}{\sqrt{K_{1}(y)}} \omega\right\|_{L^{2}}^{2}<\infty\right\}
$$

Clearly, $X \subset L^{2}$ and $L^{2}=X$ if $\min K_{1}>0$. The equation (4.2) can be written in a Hamiltonian form

$$
\omega_{t}=-U^{\prime \prime}(y) \partial_{x}\left(\frac{\omega}{K_{1}(y)}+\psi\right)=J L \omega
$$

where

$$
J=-U^{\prime \prime}(y) \partial_{x}: X^{*} \rightarrow X, \quad L=\frac{1}{K_{1}(y)}+(-\Delta)^{-1}: X \rightarrow X^{*},
$$


are anti-selfadjoint and self-adjoint respectively. Moreover, $L$ is uniformly positive on $X$ and thus $J L$ is anti-selfadjoint in the equivalent inner product $\langle L \cdot, \cdot\rangle$. Since $X \subset L^{2}$, by Lemma $5.1, J L$ has no purely imaginary eigenvalues in $X$ and the entire spectrum of $J L$ is continuous.

Case 2: Assume that there exists $U_{s}$ in the range of $U$ such that

$$
K_{2}(y)=-\frac{U^{\prime \prime}}{U-U_{s}}>0
$$

is bounded. We call these flows to be in class $\mathcal{K}^{+}$, as used in [10]. The assumption (4.3) implies that $U_{s}$ is the only inflection value of $U$. Examples include $U(y)=\sin y$, $\tanh y$, and more generally any $U(y)$ satisfying the ODE $U^{\prime \prime}=g(U)$ with a decreasing $g$. Then (4.3) is satisfied with $U_{s}=$ $g^{-1}(0)$. Let the $x$ period be $2 \pi / \alpha$. We can consider the class $\mathcal{K}^{+}$flows in a periodic channel $S_{2 \pi / \alpha} \times\left[y_{1}, y_{2}\right]$ or on a torus $S_{2 \pi / \alpha} \times S_{y_{2}-y_{1}}$. The linearized Euler equation (4.2) with $U_{s}$ being the inflection value can be written in the Hamiltonian form

$$
\omega_{t}=U^{\prime \prime}(y) \partial_{x}\left(\frac{\omega}{K_{2}(y)}-\psi\right)=J L \omega
$$

where

$$
J=U^{\prime \prime}(y) \partial_{x}, \quad L=\frac{1}{K_{2}(y)}-(-\Delta)^{-1} .
$$

Define the non-shear space of vorticity

$$
X=\left\{\omega=\sum_{k \in \mathbf{Z}, k \neq 0} e^{i k \alpha x} \omega_{k}(y),\|\omega\|_{X}^{2}=\left\|\frac{1}{\sqrt{K_{2}(y)}} \omega\right\|_{L^{2}}^{2}<\infty\right\} .
$$

Again, $X \subset L^{2}$ and $L^{2}=X$ if $\min K_{2}>0$. Denote $n^{-}(L)\left(n^{0}(L)\right)$ to be the number of negative (zero) directions of $L$ on $X$. Define the operator

$$
A_{0}=-\Delta-K_{2}(y): H^{2} \rightarrow L^{2}
$$

in the channel $S_{2 \pi / \alpha} \times\left[y_{1}, y_{2}\right]$ or on the torus $S_{2 \pi / \alpha} \times S_{y_{2}-y_{1}}$ and

$$
L_{0}=-\frac{d^{2}}{d y^{2}}-K_{2}(y): H^{2}\left(y_{1}, y_{2}\right) \rightarrow L^{2}\left(y_{1}, y_{2}\right)
$$


with the Dirichlet boundary conditions for the channel and periodic boundary conditions for the torus. Then by Lemma 11.3 in [15], we have

$$
n^{0}(L)=n^{0}\left(A_{0}\right)=2 \sum_{k \geq 1} n^{0}\left(L_{0}+k^{2} \alpha^{2}\right),
$$

and

$$
n^{-}(L)=n^{-}\left(A_{0}\right)=2 \sum_{k \geq 1} n^{-}\left(L_{0}+k^{2} \alpha^{2}\right) .
$$

If $n^{-}\left(L_{0}\right) \neq 0$, let $-\alpha_{\max }^{2}$ be the smallest eigenvalue of $L_{0}$ and $\phi_{0}$ be an eigenfunction. When $L_{0} \geq 0$, let $\alpha_{\max }=0$. Then by the above relations, $L$ is positive when $\alpha>\alpha_{\max }$. Again, by Lemma 5.1, the spectrum of $J L$ is purely continuous in $X$. When $\alpha=\alpha_{\max }$, we have $n^{-}(L)=0$ and

$$
\operatorname{ker} L=\left\{e^{ \pm i \alpha x} \omega_{0}(y)\right\}, \omega_{0}(y)=\left(-\frac{d^{2}}{d y^{2}}+\alpha^{2}\right) \phi_{0} \text {. }
$$

Let $P_{1}$ be the projection of $X$ to $\operatorname{ker} L$ and $X_{1}=\left(I-P_{1}\right) X$. Then $\left.L\right|_{X_{1}}>0$ and $A_{1}=\left(I-P_{1}\right) J L$ is anti-selfadjoint on $\left(X_{1},\langle L \cdot, \cdot\rangle\right)$.

Lemma $4.1 A_{1}$ has purely continuous spectrum on $X_{1}$.

Proof. By Lemma 3.5 of [10], $\phi_{0} \neq 0$ at at least one of the points in the set $\left\{U=U_{s}\right\}$. By using this fact, the rest of the proof follows that of Lemma 2.7, so we skip the details.

By the above spectral properties, the following is a direct consequence of the RAGE Theorem.

Theorem 4.1 If i) $U^{\prime \prime} \neq 0$ or ii) $U$ is in class $\mathcal{K}^{+}$and $\alpha>\alpha_{\max }$, then for any compact operator $B$ on $X$, we have

$$
\frac{1}{T} \int_{0}^{T}\left\|B e^{i t J L} \omega\right\|_{X}^{2} d t \rightarrow 0, \text { when } T \rightarrow \infty,
$$

for any $\omega \in X$. If $U$ is in class $\mathcal{K}^{+}$and $\alpha=\alpha_{\max }$, then for any compact operator $B$ on $X$, we have

$$
\frac{1}{T} \int_{0}^{T}\left\|B\left(I-P_{1}\right) e^{i t J L} \omega\right\|_{X}^{2} d t \rightarrow 0, \text { when } T \rightarrow \infty,
$$

for any $\omega \in X$. 
By choosing

$$
B \omega=\nabla^{\perp}(-\Delta)^{-1} \omega=u,
$$

that is, the mapping from vorticity to velocity, we get

Corollary 4.1 i) If a) $U^{\prime \prime} \neq 0$ or b) $U$ is in class $\mathcal{K}^{+}$and $\alpha>\alpha_{\max }$, then

$$
\frac{1}{T} \int_{0}^{T}\|u(t)\|_{L^{2}}^{2} d t \rightarrow 0, \text { when } T \rightarrow \infty
$$

for any solution $\omega(t)$ of (4.1) with $\omega(0) \in X$.

ii) If $U$ is in class $\mathcal{K}^{+}$and $\alpha=\alpha_{\max }$, then

$$
\frac{1}{T} \int_{0}^{T}\left\|u_{1}(t)\right\|_{L^{2}}^{2} d t \rightarrow 0, \text { when } T \rightarrow \infty
$$

where $u_{1}(t)$ is the velocity corresponding to the vorticity $\left(I-P_{1}\right) \omega(t)$ with $\omega(0) \in X$.

Remark 4.1 More information on the decay of $\|u(t)\|_{L^{2}}$ could be obtained by studying the regularity of the spectral measure of the anti-selfadjoint operator $J L$ on $(X,\langle L \cdot, \cdot\rangle)$. In particular, if the spectral measure of $J L$ is absolutely continuous (i.e. absence of singular continuous spectrum), then when $t \rightarrow \infty, \omega(t) \rightarrow 0$ weakly in $X$. As a result, $\|u(t)\|_{L^{2}} \rightarrow 0$ when $t \rightarrow \infty$.

Remark 4.2 Let $B=P_{N}$ in (4.8), i.e., the projection operator to the first $N$ Fourier modes (in $x$ ), then

$$
\frac{1}{T} \int_{0}^{T}\left\|P_{N} \omega(t)\right\|_{L^{2}}^{2} d t \rightarrow 0, \text { when } T \rightarrow \infty .
$$

This shows that in the time averaged sense, the low frequency part of $\omega$ tends to zero. As can be seen in the proof of Theorems 1.1 and 1.2, this property plays an important role in the proof of metastability of Kolmogorov flows. In the fluid literature (see e.g. [20] [5]), a dual cascade was known for 2D turbulence that energy moves to low frequency end and the enstrophy (i.e. $\left.\|\omega\|_{L^{2}}^{2}\right)$ moves to the high frequency end. The result (4.9) can be seen as a justification of such physical intuition in a weak sense. 
Remark 4.3 The two classes of shear flows considered above are linearly stable in the $L^{2}$ norm of vorticity (assuming inf $K_{i}>0$ ), in the Liapunov sense. This follows directly from the conservation of $\langle L \omega, \omega\rangle$ for the linearized Euler equation (4.2) and the positivity of $\left.L\right|_{X}$. Moreover, these two classes seem to exhaust all the possible shear flows for which nonlinear stability could be proved by the energy-Casimir method initiated by Arnold ([1] [2]) in 1960s. We briefly discuss it below and refer to [16] for more discussions on energyCasimir method for 2D Euler equations. The energy-Casimir functional is of the form

$$
H(\omega)=\int\left(G(\omega)+\frac{1}{2}|\nabla \psi|^{2}\right) d x d y,
$$

which is invariant for the nonlinear Euler equation. Suppose $\psi_{0}=F\left(\omega_{0}\right)$, where $\psi_{0}=\int\left(U-U_{s}\right) d y$ and $\omega_{0}=-U^{\prime \prime}$. Choose $G$ such that $G^{\prime}\left(\omega_{0}\right)=$ $-F\left(\omega_{0}\right)$, then $H^{\prime}\left(\omega_{0}\right)=0$ and the second order variation is given by

$$
\left\langle H^{\prime \prime}(\omega) \delta \omega, \delta \omega\right\rangle=\frac{1}{2} \int \frac{(\delta \omega)^{2}}{K_{1}(y)}+\frac{1}{2}|\nabla \delta \psi|^{2}=\frac{1}{2}\langle L \delta \omega, \delta \omega\rangle
$$

for $U(y)$ in class 1 and

$$
\left\langle H^{\prime \prime}(\omega) \delta \omega, \delta \omega\right\rangle=\frac{1}{2} \int-\frac{(\delta \omega)^{2}}{K_{2}(y)}+\frac{1}{2}|\nabla \delta \psi|^{2}=-\frac{1}{2}\langle L \delta \omega, \delta \omega\rangle
$$

for $U(y)$ in class 2. In the above, we use the relation

$$
G^{\prime \prime}\left(\omega_{0}\right)=-F^{\prime}\left(\omega_{0}\right)=\frac{U-U_{s}}{U^{\prime \prime}} .
$$

Thus $\left\langle H^{\prime \prime}(\omega) \delta \omega, \delta \omega\right\rangle$ on $X$ is positive definite for class 1 and negative definite for class 2 when $\alpha>\alpha_{\max }$. Then nonlinear stability (in $L^{2}$ vorticity) could be proved by properly handling the higher order terms. However, if $\frac{U-U_{s}}{U^{\prime \prime}}$ (equivalently $K_{1}, K_{2}$ ) changes sign, then the quadratic form $\left\langle H^{\prime \prime}(\omega) \delta \omega, \delta \omega\right\rangle$ is highly indefinite and the energy-Casimir method does not work. Despite their above restrictions, the steady flows whose stability can be studied by energy-Casimir method do appear often as observable coherent states in $2 D$ turbulence.

\subsection{Unstable case}

The shear flows $U(y)$ in class $\mathcal{K}^{+}$are proved to be linearly unstable when the horizontal wave number $\alpha<\alpha_{\max }$, see Theorem 1.2 in [10]. In this 
subsection, we will prove the inviscid damping on the center space which is complementary to the stable and unstable subspaces. The proof of linear instability in [10] is by studying the possible neutral limiting modes and the bifurcation of unstable modes near them. By using the Hamiltonian structure of (4.2) and the instability index formula in [15], we can recover this linear instability criterion. Moreover, we get more detailed information about the number of unstable modes which is important to study the inviscid damping on the center space.

Proposition 4.1 Consider $U(y)$ in class $\mathcal{K}^{+}$and $\alpha>0$, where $2 \pi / \alpha$ is the $x$ period. Let $k_{u}$ be the total algebraic multiplicities of unstable eigenvalues of the operator $J L$ defined in (4.4). Then $k_{u}=n^{-}\left(A_{0}\right)$, where $A_{0}$ is defined in (4.6).

Proof. It is easy to see that the unstable eigenfunctions of $J L$ are in the space $X$ defined in (4.5). Since on the energy space $X, n^{-}(L)=n^{-}\left(A_{0}\right)<$ $\infty$, we can use Theorem 2.3 in [15] to get the index formula

$$
k_{r}+2 k_{c}+2 k_{i}^{\leq 0}+k_{0}^{\leq 0}=n^{-}(L) .
$$

Here, $k_{r}$ and $k_{c}$ are the algebraic multiplicities of unstable eigenvalues of $J L$ lying on the positive axis and the first quadrant respectively, $k_{i}^{\leq 0}$ is the number of non-positive directions of $L$ restricted to the generalized eigenspace of imaginary eigenvalues on $i \mathbf{R}^{+}$, and $k_{0}^{\leq 0}$ is the number of non-positive directions of $L$ on $E_{0} / \operatorname{ker} L$ where $E_{0}$ is the generalized zero eigenspace of $J L$. Since $J L$ has no nonzero imaginary eigenvalue, so $k_{i}^{\leq 0}=0$. As in the proof of Lemmas 2.7 and 4.1, it can be shown that $E_{0}=\operatorname{ker} L$ and thus $k_{0}^{\leq 0}=0$. Therefore (4.10) implies that

$$
k_{u}=k_{r}+2 k_{c}=n^{-}(L)=n^{-}\left(A_{0}\right) .
$$

The space $X$ has an invariant decomposition $X=\cup_{l \in \mathbf{Z}, l \neq 0} X^{l}$, where

$$
X^{l}=\left\{e^{i \alpha l x} \omega_{l}(y), \omega_{l} \in L_{\frac{1}{K_{2}(y)}}^{2}\left(y_{1}, y_{2}\right) \cdot\right\} .
$$

On the subspace $X^{l}$, the operator $J L$ is reduced to an ODE operator $J_{l} L_{l}$ acting on the weighted space $L_{\frac{1}{K_{2}(y)}}^{2}\left(y_{1}, y_{2}\right)$, where

$$
J_{l}=U^{\prime \prime}(y) i \alpha l, \quad L_{l}=\frac{1}{K_{2}(y)}-\left(-\frac{d^{2}}{d y^{2}}+\alpha^{2} l^{2}\right)^{-1} \text {. }
$$


We have a similar counting formula for unstable eigenvalues of $J_{l} L_{l}$.

Proposition 4.2 Consider $U(y)$ in class $\mathcal{K}^{+}$and $\alpha>\alpha_{\max }$. Let $k_{u, l}$ be the total algebraic multiplicities of the unstable eigenvalues of the operator $J_{l} L_{l}(0 \neq l \in \mathbf{Z})$ defined in (4.11). Then

$$
k_{u, l}=n^{-}\left(L_{0}+l^{2} \alpha^{2}\right),
$$

where $L_{0}$ is defined in (4.7).

Proof. Since $J_{l}$ is not a real operator, we can not directly apply the index Theorem 2.3 in [15]. Define the space

$$
\begin{aligned}
Y_{l} & =X^{l} \oplus X^{-l} \\
& =\left\{\cos (\alpha l x) \omega_{1}(y)+\sin (\alpha l x) \omega_{2}(y), \omega_{1}, \omega_{2} \in L_{\frac{1}{K_{2}(y)}}^{2}\left(y_{1}, y_{2}\right)\right\},
\end{aligned}
$$

which is isomorphic to the space $Y=\left(L_{\frac{1}{K_{2}(y)}}^{2}\left(y_{1}, y_{2}\right)\right)^{2}$. For any

$$
\omega=\cos (\alpha l x) \omega_{1}(y)+\sin (\alpha l x) \omega_{2}(y) \in Y_{l},
$$

we have

$$
J L \omega=(\cos (\alpha l x), \sin (\alpha l x)) J^{l} L^{l}\left(\begin{array}{l}
\omega_{1} \\
\omega_{2}
\end{array}\right)
$$

where

$$
J^{l}=\left(\begin{array}{cc}
0 & \alpha l U^{\prime \prime} \\
-\alpha l U^{\prime \prime} & 0
\end{array}\right), \quad L^{l}=\left(\begin{array}{cc}
L_{l} & 0 \\
0 & L_{l}
\end{array}\right) .
$$

In the above, the operator $L_{l}$ is defined in (4.11). Thus to study $J L$ on $Y_{l}$, it is equivalent to study $J^{l} L^{l}$ on $Y$. Let $k_{u}^{l}$ be the total algebraic multiplicities of the unstable eigenvalues of the operator $J^{l} L^{l}$. Then by Theorem 2.3 in [15] and the same proof of Proposition 4.2, we have

$$
k_{u}^{l}=n^{-}\left(L^{l}\right)=2 n^{-}\left(L_{l}\right)=2 n^{-}\left(L_{0}+l^{2} \alpha^{2}\right) .
$$

Since the spectra of $J_{l} L_{l}$ is complex conjugate of that of $J_{-l} L_{-l}$, so

$$
k_{u}^{l}=k_{u, l}+k_{u,-l}=2 k_{u, l}
$$

and this finishes the proof of the proposition. 
Remark 4.4 Let $\lambda=i \alpha l c$ be an eigenvalue of $J_{l} L_{l}$ and $J_{l} L_{l} \omega=\lambda \omega$ for some $0 \neq \omega \in X^{l}$. Then the stream function

$$
\psi(y)=\left(-\frac{d^{2}}{d y^{2}}+\alpha^{2} l^{2}\right)^{-1} \omega(y)
$$

satisfies the classical Rayleigh equation

$$
\left(-\frac{d^{2}}{d y^{2}}+\alpha^{2} l^{2}+\frac{U^{\prime \prime}}{U-c}\right) \psi=0
$$

with Dirichlet or periodic boundary conditions. It was shown in [8] that for $U(y)$ in class $\mathcal{K}^{+}$, the total number (i.e. geometric multiplicities) of unstable eigenvalues (i.e. $\operatorname{Im} c>0)$ can not exceed $n^{-}\left(L_{0}+l^{2} \alpha^{2}\right)$. In [10], it was shown that $k_{u, l} \geq 1$ when $n^{-}\left(L_{0}+l^{2} \alpha^{2}\right) \neq 0$. The precise index formula (4.12) not only gives an improvement over previous results, but also is important below for understanding the dynamics on the center space.

Denote $E^{s}\left(E^{u}\right) \subset X$ to be the stable (unstable) eigenspace of $J L$, then

$$
\operatorname{dim} E^{s}=\operatorname{dim} E^{u}=k_{u}=n^{-}(L) .
$$

Moreover, by Corollary 6.1 in [15], $\left.L\right|_{E^{s} \oplus E^{u}}$ is non-degenerate and

$$
n^{-}\left(E^{s} \oplus E^{u}\right)=\operatorname{dim} E^{u}=n^{-}(L) .
$$

Define the center space $E^{c}$ to be the orthogonal (in the inner product $\langle L \cdot, \cdot\rangle$ ) complement of $E^{s} \oplus E^{u}$ in $X$, that is,

$$
E^{c}=\left\{\omega \in X \mid\left\langle L \omega, \omega_{1}\right\rangle=0, \forall \omega_{1} \in E^{s} \oplus E^{u}\right\} .
$$

Then we have

Lemma 4.2 The decomposition $X=E^{s} \oplus E^{u} \oplus E^{c}$ is invariant under $J L$. Moreover, $n^{-}\left(\left.L\right|_{E^{c}}\right)=0$ and as a consequence $\left.L\right|_{E^{c} / \operatorname{ker} L}>0$.

Proof. The invariance of the decomposition follows from the invariance of $\langle L \cdot, \cdot\rangle$ under $J L$. By (4.14), we have

$$
n^{-}\left(\left.L\right|_{E^{c}}\right)=n^{-}(L)-n^{-}\left(E^{s} \oplus E^{u}\right)=0,
$$

and thus $\left.L\right|_{E^{c} / \operatorname{ker} L}>0$.

Since $E^{c}$ is invariant under $J L$, we can restrict the linearized Euler equation on $E^{c}$. The linear inviscid damping still holds true for initial data in $E^{c}$. Denote $P_{1}$ to be the projection of $X$ to ker $L$. By the same proof of Theorem 4.1 and Corollary 4.1, we have the following. 
Theorem 4.2 Suppose $U(y)$ is in class $\mathcal{K}^{+}$and $\alpha<\alpha_{\max }$.

i) If $\operatorname{ker} L=\{0\}$, then

$$
\frac{1}{T} \int_{0}^{T}\|u(t)\|_{L^{2}}^{2} d t \rightarrow 0, \text { when } T \rightarrow \infty
$$

for any solution $\omega(t)$ of $(4.1)$ with $\omega(0) \in E^{c}$. Here, $E^{c}$ is the center space defined in (4.15).

ii) If $\operatorname{ker} L \neq\{0\}$, then

$$
\frac{1}{T} \int_{0}^{T}\left\|u_{1}(t)\right\|_{L^{2}}^{2} d t \rightarrow 0, \text { when } T \rightarrow \infty
$$

where $u_{1}(t)$ is the velocity corresponding to the vorticity $\left(I-P_{1}\right) \omega(t)$ with $\omega(0) \in E^{c}$.

Remark 4.5 Above theorem suggests that the dynamics of solutions of the linearized Euler equation on the center space $E^{c}$ is similar to the stable case.

The invariant decomposition $X=E^{s} \oplus E^{u} \oplus E^{c}$ can be used to prove the exponential trichotomy of the semigroup $e^{t J L}$. We refer to Theorem 2.2 in [15] for the precise statement. The next natural step is to construct invariant manifolds (stable, unstable and center) for the nonlinear Euler equation, which will give a complete description of the local dynamics near $u_{0}=(U(y), 0)$. The stable and unstable manifolds near any unstable shear flow were constructed in [14]. The construction of center manifold is under investigation. Once constructed, on such center manifold, the positivity of $\left.L\right|_{E^{c}}$ (Lemma 4.2) could be used to prove nonlinear stability of solutions.

\section{Appendix}

Lemma 5.1 Let $U(y) \in C^{2}\left(y_{1}, y_{2}\right)$, where $-\infty<y_{1}<y_{2}<\infty$. Consider the Rayleigh equation

$$
\left(-\frac{d^{2}}{d y^{2}}+\alpha^{2}+\frac{U^{\prime \prime}}{U-c}\right) \psi=0,
$$

with the periodic boundary condition

$$
\psi\left(y_{1}\right)=\psi\left(y_{2}\right), \psi^{\prime}\left(y_{1}\right)=\psi^{\prime}\left(y_{2}\right)
$$


or the Dirichlet boundary condition

$$
\psi\left(y_{1}\right)=\psi\left(y_{2}\right)=0 .
$$

If (5.1) has a neutral solution with $\alpha>0, c \in \mathbf{R}$ and $\psi \in H^{2}\left(y_{1}, y_{2}\right)$, then c must be an inflection value of $U$.

Proof. First, we show that $c$ must be in the range of $U(y)$. Suppose otherwise $c>\max U$ or $c<\min U$. Assume $c>\max U$. For the Dirichlet boundary condition, since $U-c<0$ in $\left[y_{1}, y_{2}\right]$, by the proof of Lemma 3.5 of [10], $\psi \equiv 0$ in $\left[y_{1}, y_{2}\right]$, which is a contradiction. For the periodic boundary condition, (5.1) implies that the operator $L_{0}=-\frac{d^{2}}{d y^{2}}-\frac{U^{\prime \prime}}{U-c}$ has a negative eigenvalue $-\alpha^{2}$. Let $\lambda_{0} \leq-\alpha^{2}<0$ be the smallest eigenvalue of $L_{0}$, then the corresponding eigenfunction $\phi$ can be taken to be positive. The equation $L_{0} \phi=\lambda_{0} \phi$ can be written as

$$
\left((U-c) \phi^{\prime}-U^{\prime} \phi\right)^{\prime}=-\lambda_{0}(U-c) \phi .
$$

Integrating above from $y_{1}$ to $y_{2}$ and using the periodic boundary condition, we have

$$
\int_{y_{1}}^{y_{2}}(U-c) \phi d y=0,
$$

which is a contradiction again. Therefore $c$ must be in the range of $U$.

Let $z_{1}<z_{2}<\cdots<z_{k_{c}}\left(k_{c} \geq 1\right)$ be the zeros of $U(y)-c$ in $\left[y_{1}, y_{2}\right]$. We claim that there exists $1 \leq k \leq k_{c}$ such that $\psi\left(z_{k}\right) \neq 0$. For the Dirichlet boundary condition, this follows by Lemma 3.5 of [10]. For the periodic boundary condition, suppose otherwise $\psi\left(z_{k}\right)=0$ for all $k=1,2, \cdots, k_{c}$. Let $z_{k_{c}+1}=z_{1}+y_{2}-y_{1}$ which is the translation of $z_{1}$ by one period. Then $U-c$ takes the same sign on each interval $\left[z_{i}, z_{i+1}\right], i=1,2, \cdots, k_{c}$, and $\psi=0$ at the end points. By the proof of Lemma 3.5 of [10], it follows that $\psi \equiv 0$ in all the intervals $\left[z_{i}, z_{i+1}\right]$. Thus $\psi \equiv 0$ in $\left[y_{1}, y_{2}\right]$, a contradiction. Let $1 \leq k_{0} \leq k_{c}$ be such that $\psi\left(z_{k_{0}}\right) \neq 0$. Then we must have $U^{\prime \prime}\left(z_{k_{0}}\right)=0$, that is, $c=U\left(z_{k_{0}}\right)$ is an inflection value. Suppose otherwise, then $U^{\prime \prime}\left(z_{k_{0}}\right) \neq 0$ and thus $\frac{U^{\prime \prime}}{U-c} \psi$ is not in $L_{l o c}^{2}$ near $z_{k_{0}}$, which is in contradiction to the Rayleigh equation (5.1) and the assumption that $\psi \in H^{2}$. This finishes the proof of the Lemma.

Remark 5.1 The above lemma shows that for general shear flows $U(y)$, any $H^{2}$ neutral mode must have its phase speed c to be one of the inflection values. 
This fact is used in section 4 to exclude embedded eigenvalues and obtain the instability index formula (4.12) and the positivity of $\left.L\right|_{E^{c}}$. In [10] [12], it was shown for a class of shear flows (called class $\mathcal{F}$ in [10]) that any neutral limiting mode (i.e. the limit of a sequence of unstable modes) must be in $\mathrm{H}^{2}$ and therefore the phase speed must be inflection values. These neutral limiting modes are important for finding linear stability/instability criteria since they give the transition points for stability/instability.

The flows $U(y)$ in class $\mathcal{F}$ (see [10] for definition) include any monotone flow, class $\mathcal{K}^{+}$flows, and more generally, any $U(y)$ satisfying an ODE $U^{\prime \prime}=$ $k(y) g(U)$ for some $k>0$ and any $g$. However, for shear flows not in class $\mathcal{F}$, the limiting neutral modes might be singular (i.e. not in $\mathrm{H}^{2}$ ). Such singular neutral modes might have their phase speeds $c$ other than the inflection values.

\section{Acknowledgement}

Zhiwu Lin is supported in part by NSF grants DMS-1411803 and DMS1715201.

\section{References}

[1] Arnold, V. I. On conditions for non-linear stability of plane stationary curvilinear flows of an ideal fluid. (Russian) Dokl. Akad. Nauk SSSR 162 (1965), 975-978.

[2] Arnold, V. I. An a priori estimate in the theory of hydrodynamic stability. (Russian) Izv. Vysš. Učebn. Zaved. Matematika 54 (1966), 3-5.

[3] Beck, Margaret; Wayne, C. Eugene; Metastability and rapid convergence to quasi-stationary bar states for the two-dimensional Navier-Stokes equations. Proc. Roy. Soc. Edinburgh Sect. A 143 (2013), 905-927.

[4] Bedrossian, J. \& Masmoudi, N. Inviscid damping and the asymptotic stability of planar shear flows in the 2D Euler equations. Publ. Math. Inst. Hautes Études Sci. 122 (2015), 195-300.

[5] Bouchet, F., Venaille, A., Statistical mechanics of two-dimensional and geophysical flows. Phys. Rep. 5 (2012), 227-295. 
[6] Bouchet, F. and Simonnet, E., Random changes of flow topology in twodimensional and geophysical turbulence. Phys. Rev. Lett., 102 (2009), 094504 .

[7] Cycon, H. L.; Froese, R. G.; Kirsch, W.; Simon, B. Schrödinger operators with application to quantum mechanics and global geometry. Texts and Monographs in Physics. Springer-Verlag, Berlin, 1987.

[8] Howard, Louis N. The number of unstable modes in hydrodynamic stability problems. J. Mécanique 3 (1964), 433-443.

[9] Constantin, P.; Kiselev, A.; Ryzhik, L.; Zlatoš, A. Diffusion and mixing in fluid flow. Ann. of Math. (2), 168 (2008), 643-674.

[10] Lin, Zhiwu Instability of some ideal plane flows. SIAM J. Math. Anal. 35 (2003), 318-356.

[11] Lin, Zhiwu, Some stability and instability criteria for ideal plane flows, Comm. Math. Phys., 246 (2004), 87-112.

[12] Lin, Zhiwu, Some recent results on instability of ideal plane flows, Nonlinear partial differential equations and related analysis, 217-229, Contemp. Math., 371, Amer. Math. Soc., Providence, RI, 2005.

[13] Lin, Zhiwu and Zeng, Chongchun, Inviscid dynamic structures near Couette flow, Arch. Ration. Mech. Anal. 200 (2011), 1075-1097.

[14] Lin, Zhiwu and Zeng, Chongchun, Unstable manifolds of Euler equations, Comm. Pure Appl. Math. 66 (2013), 1803-1936.

[15] Lin, Zhiwu and Zeng, Chongchun, Instability, index theorem, and exponential trichotomy for Linear Hamiltonian PDEs, arXiv:1703.04016.

[16] Marchioro, C. and Pulvirenti, M., Mathematical Theory of Incompressible Nonviscous Fluids, Springer-Verlag 1994.

[17] Matthaeus, W. H.; Stribling, W. T. ; Martinez, D.; Oughton, S. and Montgomery, D. Decaying, two-dimensional, Navier-Stokes turbulence at very long times. Physica D 51 (1991), 531-538.

[18] Orr, W. McF. Stability and instability of steady motions of a perfect liquid, Proc. Ir. Acad. Sect. A, Math Astron. Phys. Sci. 27 (1907), 9-66. 
[19] Robert, Raoul Statistical hydrodynamics (Onsager revisited). Handbook of mathematical fluid dynamics, Vol. II, 1-54, North-Holland, Amsterdam, 2003.

[20] Tabeling, Patrick Two-dimensional turbulence: a physicist approach. Phys. Rep. 362 (2002), 1-62.

[21] Villani, C., Hypocoercivity, Memoirs of the American Mathematical Society, American Mathematical Society, 2009.

[22] Yin, Z.; Montgomery, D. C.; Clercx, H. J. H. Alternative statisticalmechanical descriptions of decaying two-dimensional turbulence in terms of "patches" and "points". Phys. Fluids 15 (2003), 1937-1953.

[23] Wei, D., Zhang, Z. and Zhao, W., Linear Inviscid damping for a class of monotone shear flow in Sobolev spaces, Comm. Pure. Appl. Math., to appear.

[24] Wei, D., Zhang, Z. and Zhao, W., Linear Inviscid damping and vorticity depletion for shear flows, preprint.

[25] Zillinger, C. Linear inviscid damping for monotone shear flows in a finite periodic channel, boundary effects, blow-up and critical Sobolev regularity, Arch. Ration. Mech. Anal. 221 (2016), 1449-1509. 